\title{
Combination of Searches for Higgs Boson Pair Production in Proton-Proton Collisions at $\sqrt{s}=13 \mathrm{TeV}$
}

\author{
A. M. Sirunyan et al. \\ (CMS Collaboration)
}

(Received 23 November 2018; published 29 March 2019)

\begin{abstract}
This Letter describes a search for Higgs boson pair production using the combined results from four final states: $b b \gamma \gamma, b b \tau \tau, b b b b$, and $b b V V$, where $V$ represents a $W$ or $Z$ boson. The search is performed using data collected in 2016 by the CMS experiment from LHC proton-proton collisions at $\sqrt{s}=13 \mathrm{TeV}$, corresponding to an integrated luminosity of $35.9 \mathrm{fb}^{-1}$. Limits are set on the Higgs boson pair production cross section. A 95\% confidence level observed (expected) upper limit on the nonresonant production cross section is set at 22.2 (12.8) times the standard model value. A search for narrow resonances decaying to Higgs boson pairs is also performed in the mass range $250-3000 \mathrm{GeV}$. No evidence for a signal is observed, and upper limits are set on the resonance production cross section.
\end{abstract}

DOI: 10.1103/PhysRevLett.122.121803

The discovery of the Higgs boson $(H)$ by the ATLAS and CMS Collaborations [1-3] was a major step in the understanding of the mechanism of electroweak symmetry breaking. The Higgs boson mass was jointly determined by the two experiments using the CERN LHC Run 1 data to be $m_{H}=125.09 \pm 0.24 \mathrm{GeV}$ [4] and recently by CMS using partial Run 2 data with even better accuracy, $m_{H}=$ $125.26 \pm 0.21 \mathrm{GeV}$ [5]. With the Higgs boson mass known with a precision better than $0.2 \%$, the structure of the Higgs scalar field potential and the Higgs boson self-couplings are precisely predicted in the standard model (SM). The current measurements of the properties of the Higgs boson are compatible with the SM predictions [6,7]. The measurement of the Higgs boson self-coupling provides an independent test of the SM and verification that the Higgs mechanism is truly responsible for the electroweak symmetry breaking by giving access to the shape of the Higgs scalar field potential [8]. Access to the Higgs boson trilinear coupling can be obtained by measuring the production of pairs of Higgs bosons $(H H)$ at the LHC. At the same time, several theories predict heavy resonances that decay into $H H$ [9-16]. Studies of pair production of Higgs bosons, each of which can decay in different channels, allow one to probe different regions of the anomalous couplings space and of the resonant invariant mass spectrum. A combination of different channels is

\footnotetext{
*Full author list given at the end of the Letter.

Published by the American Physical Society under the terms of the Creative Commons Attribution 4.0 International license. Further distribution of this work must maintain attribution to the author(s) and the published article's title, journal citation, and DOI. Funded by SCOAP ${ }^{3}$.
}

therefore needed to obtain the best possible sensitivity for the $H H$ production.

The $H H$ SM production cross section, which partly depends on the Higgs trilinear coupling, was computed at next-to-next-to-leading order in quantum chromodynamics (QCD), including next-to-next-to-leading-logarithmic corrections and finite top quark mass effects at next-toleading order. Its value is $\sigma_{H H}=33.53_{-6.0 \%}^{+4.3 \%}(\mathrm{QCD}$ scale $) \pm$ $5.9 \%$ (other) fb in proton-proton $(p p)$ collisions at $13 \mathrm{TeV}$ for a Higgs boson mass of $125 \mathrm{GeV}$ [17-21], where "other" includes contributions from parton distribution function (PDF) uncertainties evaluated using the PDF4LHC recommendations [22-24], strong coupling constant $\alpha_{S}$ dependence, and top quark mass effects.

Physics beyond the SM (BSM) can significantly modify the cross section and the kinematic properties of nonresonant Higgs boson pair production. In order to provide model independent constraints on these effects, we introduce an effective field theory (EFT) Lagrangian that extends the SM with dimension-6 operators [25]. This approach results in five anomalous Higgs boson couplings relevant for $H H$ production: the $H$ coupling to the top quark, $y_{t}$; the trilinear coupling, $\lambda_{H H H}$; and three additional couplings, denoted by $c_{2}, c_{2 g}$, and $c_{g}$, that represent, respectively, the interactions of a top quark pair with a Higgs boson pair, of a gluon pair with a Higgs boson pair, and of a gluon pair with a single $H$ [17]. We define $k_{\lambda}=$ $\lambda_{H H H} / \lambda_{\mathrm{SM}}$ and $k_{t}=y_{t} / y_{t}^{\mathrm{SM}}$. Since a full five-dimensional scan of all possible coupling combinations would be computationally excessive, a clustering strategy [26] has been developed to group together possible combinations of coupling values that present similar kinematic properties. Twelve clusters have been identified, in addition to the $\mathrm{SM}$ and the $\lambda_{H H H}=0$ scenarios. Within each cluster, a 
representative point in the EFT space that we refer to as a benchmark is selected. Each benchmark thus represents a possible modification of the $H H$ signal yield and kinematic distributions due to BSM effects.

In the searches discussed in the Letter, the resonant signal is represented by either a $C P$-even particle of spin- 0 (radion) or spin-2 (graviton) with a width that is much smaller than the detector resolution for the whole range under study.

The ATLAS and CMS Collaborations performed studies of Higgs boson pair production at $\sqrt{s}=8 \mathrm{TeV}$ [27-29]. Limits on nonresonant $H H$ production were set by the CMS Collaboration in the $b b \gamma \gamma$ and $b b \tau \tau$ final states. Here and in the rest of the text the indication of the charge of the decay products is omitted for simplicity of notation. The combination of those searches allowed the CMS Collaboration to set an upper limit on $H H$ production at 43 times the SM expectation [29]. Using the data collected in 2016 at $\sqrt{s}=13 \mathrm{TeV}$, the CMS and ATLAS Collaborations performed searches in the $b b \gamma \gamma$ [30,31], $b b \tau \tau$ [32,33], and bbbb [34-38] final states, with the CMS Collaboration having results in the $b b V V$ [39] channel as well, where $V$ denotes either a $W$ or a $Z$ boson that decays leptonically. All four channels studied at CMS are included in this combination.

The CMS apparatus features a superconducting solenoid of $6 \mathrm{~m}$ internal diameter, providing a magnetic field of $3.8 \mathrm{~T}$. Within the solenoid volume are a silicon pixel and strip tracker, a lead tungstate crystal electromagnetic calorimeter, and a brass and scintillator hadron calorimeter, each composed of a barrel and two end cap sections. Forward calorimeters extend the pseudorapidity coverage provided by the barrel and end cap detectors. Muons are detected in gas-ionization chambers embedded in the steel flux-return yoke outside the solenoid. Events of interest are selected using a two-tiered trigger system [40]. The particle-flow algorithm [41] aims to reconstruct and identify each individual particle in an event with an optimized combination of information from the various elements of the CMS detector. Dedicated $b$ tagging algorithms [42] are used to identify jets originating from $b$ quarks ( $b$ jets). A more detailed description of the CMS detector, together with a definition of the coordinate system used and the relevant kinematic variables, can be found in Ref. [43].

With the current data, the $b b \gamma \gamma$ [30] analysis is the most sensitive to SM Higgs boson pair production in CMS. Despite a low branching fraction $[\mathcal{B}(H H \rightarrow b b \gamma \gamma)=$ $0.26 \%$, the analysis profits from a very small background contribution, thanks to the excellent diphoton mass resolution of the CMS experiment for this channel $(\approx 1.6 \mathrm{GeV}$ [30]). To exploit this feature, the analysis relies on a $2 \mathrm{D}$ fit of the $H \rightarrow \gamma \gamma$ and $H \rightarrow b b$ invariant mass distributions, where the background coming from the $\mathrm{N} \gamma+$ jets $(N=$ $\{0,1,2, \ldots\})$ continuum is estimated from the mass sidebands. Other background contributions can arise from single $H$ production and constitute up to a third of the total background in the nonresonant searches. They are modeled from Monte Carlo (MC) simulations, including gluon fusion, vector boson fusion, $V H, t \bar{t} H$, and $b \bar{b} H$ production processes. In the nonresonant searches events are classified into low- and high-mass categories according to the $H H$ pair reduced mass $\tilde{M}_{\mathrm{X}}=M_{\mathrm{jj} \gamma \gamma}-$ $M_{\mathrm{jj}}-M_{\gamma \gamma}+250 \mathrm{GeV}$, which increases the sensitivity to $H H$ production. A further categorization based on the purity of the events is applied to both resonant and nonresonant searches. The signal purity is estimated by means of a boosted decision tree (BDT) built from the $b$ tagging probability of each jet, the angles between the products of the $\mathrm{HH}$ system decay, and the transverse momentum of each $H$ candidate. The category most sensitive to nonresonant production is the one with the highest signal purity and reduced mass, with a SM signal over background ratio $(S / B)$ of $\approx 5 \%$. The low reduced mass categories enhance sensitivity to large $k_{\lambda}$ values. In the resonant search, two different BDTs are trained for resonance masses $m_{\mathrm{X}}$ higher or lower than $600 \mathrm{GeV}$.

The $b b \tau \tau$ analysis [32] combines a relatively low background contamination with a relatively large branching fraction $(7.3 \%)$, for a final $S / B$ of $\approx 0.4 \%$ in the most sensitive category. At least one isolated hadronically decaying $\tau$ lepton must be present in the event, together with a second isolated lepton that is oppositely charged. The second lepton can be either an electron or a muon (semileptonic final state) or another hadronically decaying $\tau$ lepton (fully hadronic final state). Events are categorized according to the number of $b$-tagged jets (one or two) in the event. Events with a boosted $H$ jet candidate are assigned to a dedicated boosted category. A BDT discriminant based on the kinematic differences between the $H H$ and $t \bar{t}$ processes is used in the semileptonic channels in order to reduce the large amount of $t \bar{t}$ background. A kinematic fit [44] is performed to reconstruct the Higgs boson mass. For the nonresonant searches, the so-called "stransverse mass" $m_{\mathrm{T} 2}[32,45,46]$ is found to provide the best separation between the background and the signal [45]. For the resonant searches, a further kinematic fit, detailed in Ref. [47], is performed to reconstruct the most probable mass of the resonance. The largest background contributions come from Drell-Yan, $t \bar{t}$, processes and events comprised uniquely of jets produced via the strong interaction (QCD multijet). Additional background sources considered are $V H, V V$, and $W+$ jets. The DrellYan background is estimated from a leading order simulation with a scale factor obtained from data in a $Z$ boson enriched control region to account for higherorder corrections. The QCD multijet background is estimated using three control regions with different lepton charge (same-sign region) and isolation requirements. All other background contributions are estimated from $\mathrm{MC}$ simulations. 
The $b b b b$ final state has the highest $H H$ decay branching fraction $(33.6 \%)$. Three optimized resonance searches are performed, targeting resonances of different masses. For a resonance mass below $0.7 \mathrm{TeV}$, four $b$-tagged jets [34] are required in the final state. For high-mass searches $\left(m_{\mathrm{X}}>1.2 \mathrm{TeV}\right)$, selected events must contain two " $H$ jet candidates," where each $H$ jet candidate is a jet associated with a boosted $H$ decaying into a $b \bar{b}$ pair merged into a single jet [35]. This candidate reconstruction uses jet substructure and jet flavor tagging techniques [48]. The search sensitivity in the intermediate mass range, 0.71.2 TeV, is improved by considering both the final state with four $b$ jets and the case with one Higgs jet candidate and two $b$ jets [36]. A dedicated nonresonant $H H$ search is performed in the four $b$ jet final state [37]. Triggers using $b$ tagging and jet substructure techniques are used to collect these events. The sensitivity of the analysis is improved using a BDT that employs jet-related and $H H$ decay kinematic variables and global event properties. Under SM assumptions, the $S / B$ can be as high as $1 \%$ in the most sensitive BDT bins. Further sensitivity to BSM nonresonant $H H$ production, which often results in boosted topologies, is obtained adding the final states with one or two $H$ jet candidates [36]. The dominant background comes from QCD multijet events and it is estimated using data sideband regions [34-36] and a hemisphere mixing technique [37]. The residual background is dominated by $t \bar{t}$ events and is estimated using simulation. Such events correspond to 10,15 , and $1 \%$ of the total background in the low-, intermediate-, and high-mass ranges, respectively, for resonant searches and 5 (15)\% for nonresonant searches in the final states with four $b$-tagged jets (with $H$ jet candidates).

The $b b V V$ analysis [39] includes the $H H \rightarrow b b W W \rightarrow$ $b b \ell \nu \ell \nu$ and the $H H \rightarrow b b Z Z \rightarrow b b \ell \ell \nu \nu$ processes, for a total branching fraction $\mathcal{B}(H H \rightarrow b b \ell \nu \ell \nu)=2.72 \%$ when all possible lepton flavor decays are considered (including the $\tau$ leptonic decays). This channel has large backgrounds, which are predominantly from $t \bar{t}$ and Drell-Yan processes. All background sources are estimated from MC simulations with the exception of Drell-Yan, which is estimated from data. In order to improve the rejection of these large backgrounds, a deep neural network (DNN) discriminant technique has been implemented. Two separate parametrized DNNs have been trained, one for the resonant and one for the nonresonant search. The first is parametrized according to the mass of the resonance, while the second depends on the parameters $k_{\lambda}$ and $k_{t}$. For each nonresonant benchmark, the closest point in the $\left(k_{\lambda}, k_{t}\right)$ plane, according to the statistical measure defined in Ref. [26], has been used to define the DNN parameters. An $S / B$ ratio of $\approx 0.18 \%$ is obtained in the most sensitive DNN region for SM nonresonant production.

For both the resonant and nonresonant searches, likelihood fits are performed using the statistical toolkits,
ROOFIT [49] and ROOSTATS [50]. The signal strength $(\mu)$, defined as the ratio between the observed and expected signal rates, is estimated with its corresponding confidence interval via the profile likelihood ratio test statistic [51]. The latter depends on the signal strength as well as on the nuisance parameters, which account for various experimental and theoretical uncertainties. The reference value for the expected signal strength is chosen as the SM gluon fusion $\mathrm{HH}$ production cross section (33.53 fb) in nonresonant searches. The likelihood fits are performed with respect to the observed data or a data set constructed using $\mu=1$ for assessing expected results using the asymptotic approximation [52,53]. Limits are set at 95\% confidence level (C.L.) using the $\mathrm{CL}_{\mathrm{s}}$ criterion [54,55]. For all measurements, the $H$ mass is fixed at $m_{H}=125 \mathrm{GeV}$, and branching fractions are assumed to be equal to the SM predictions. When investigating signal models corresponding to the shape benchmarks, the single $H$ production cross sections are all assumed to have their SM values.

When combining results, the systematic uncertainties from various sources are accounted for as follows. A polynomial interpolation between alternative shape variations is used to model systematic effects on the shape of the discriminant variables. Lepton and photon reconstruction and identification efficiencies and energy scale corrections are assumed to be fully correlated across the channels and analysis categories that use the same objects. The uncertainties in the jet energy scale corrections are divided into multiple sources. The effect of each source on the rate and shape of the final observable for different processes is considered in the $b b V V$ analysis and is treated as fully correlated with the normalization effects in the $b b \tau \tau$ and $b b \gamma \gamma$ channels. In these latter channels, the shape effects from the individual sources have been verified to be negligible, and only the cumulative effects on the shape are considered. They are assumed to be correlated with the normalization and shape variation from the $b b b b$ analysis. Jet energy resolution effects are negligible in the $b b \tau \tau$ channel and assumed to be fully correlated between the $b b V V$ and the $b b b b$ channels. The effects of jet energy scale and resolution are included in the functional form for the shape used in the bby channel and are assumed to be uncorrelated with the other channels. Uncertainties related to the $b$ tagging are dominated by the modeling of heavy flavor production when measuring data/MC scale factors, and are considered correlated across the $b b \tau \tau, b b \gamma \gamma$, resonant $b b b b$, and $b b V V$ channels. The nonresonant $b b b b$ analysis uses a different $b$ tagging method and is considered uncorrelated.

An integrated luminosity uncertainty of $2.5 \%$ [56] is applied in a fully correlated way to all channels and all processes estimated from simulation. Different uncertainties are applied to background processes that are estimated from data. The uncertainties in the total cross sections of the common background processes are assumed to be 
correlated across all channels, and are of the order of $5 \%$ for the most relevant ones (single top, $t \bar{t}, V H$ ). The uncertainty in the same-sign to opposite-sign candidate ratio in the $b b \tau \tau$ channel is propagated to the estimation of the multijet background, and the uncertainties in the scale factors applied to the $Z / \gamma^{*} \rightarrow \ell \ell$ background estimation to correct for higher-order effects are also taken into account. A normalization uncertainty is considered in the $b b b b$ nonresonant search to account for residual biases in the hemisphere mixing technique. Some background estimates in the $b b \tau \tau, b b V V$, and nonresonant $b b b b$ analyses have non-negligible statistical uncertainties due to the limited number of events passing the selection in data control regions or simulated data samples. These are taken into account by allowing each bin in each template shape to fluctuate independently according to a Poisson distribution. These uncertainties are assumed to be uncorrelated across bins in the individual template shapes. The nonresonant signal uncertainties include contributions coming from variations in the renormalization and factorization scales, these amount to ${ }_{-6.0 \%}^{+4.3 \%}[17,20]$ of the nonresonant signal cross section. Other theoretical uncertainties such as those in $\alpha_{S}$, PDFs, and finite top quark mass effects at next-tonext-to-leading order result in a further $5.9 \%$ uncertainty $[18,19,21]$. These effects are assumed to be fully correlated across the different channels. The $\alpha_{S}$, PDFs, and scale variation effects are also included for single $H$ background contributions. These uncertainties are considered fully correlated for the $V H$ production in the $b b \tau \tau, b b V V$, and $b b \gamma \gamma$ channels. The uncertainty in the branching fraction of the Higgs boson to $b b$ [17] is also assumed to be fully correlated across all channels.

The event yield in data is small, so the statistical uncertainties are much larger than the systematic ones. Those with the largest effect on the final result are the statistical fluctuations in the yield in the most sensitive bins of the BDT and the overall background normalization in the $b b b b$ channel, the hadronically decaying $\tau$ lepton energy scale effects in the $b b \tau \tau$ analysis, and the uncertainty in the functional form used to model the signal shape in the $b b \gamma \gamma$ channel. These effects are as large as $10(5) \%$ for the $b b b b$ and $b b \tau \tau(b b \gamma \gamma)$ uncertainties. Due to its lower overall sensitivity, the systematic uncertainties affecting the $b b V V$ analysis have little effect on the combined result. The largest sources of systematic uncertainty for this channel arise from the uncertainties in the $t \bar{t}$ cross section, electron identification efficiency, and $b$ tagging efficiency.

With all the correlations across the channels included, the observed and expected limits at $95 \%$ confidence level on the nonresonant $H H$ production signal strength are measured to be 22.2 and 12.8 times the SM expectations, respectively. They are shown in Fig. 1 for the individual channels and their combination. Small excesses, compatible with statistical fluctuations, are observed in the $b b b b$, $b b \tau \tau$, and $b b \gamma \gamma$ final states and result in a small excess in

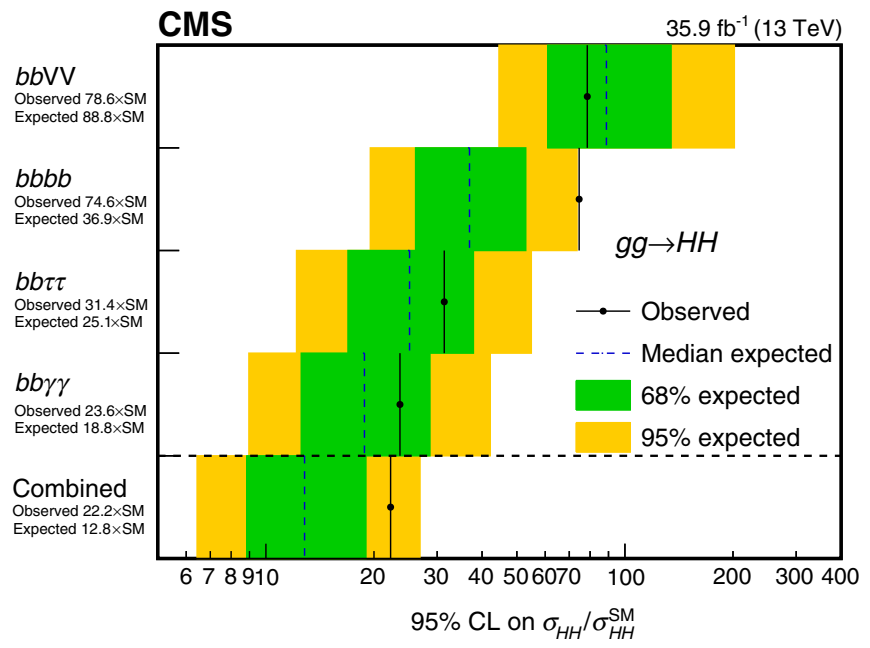

FIG. 1. The 95\% C.L. upper limits on the signal strength $\mu=\sigma_{H H} / \sigma_{H H}^{\mathrm{SM}}$. The inner (green) band and the outer (yellow) band indicate the regions containing 68 and 95\%, respectively, of the limits on $\mu$ expected under the background-only hypothesis.

the combined result. A scan is performed for different values of the $k_{\lambda}$ parameter, while keeping all other EFT parameters fixed at their SM values. The value of $k_{\lambda}$ affects both the expected cross section and the $H H$ decay kinematics. For each value, these differences are fully simulated and considered in the scan. Resulting limits are reported in Fig. 2. The exclusion limit as a function of $k_{\lambda}$ closely follows the features of the $H H$ production cross section and $H H$ invariant mass distribution $M_{H H}$ [57], which are sculpted by the interference between the $\mathrm{HH}$ production via the trilinear Higgs coupling and the

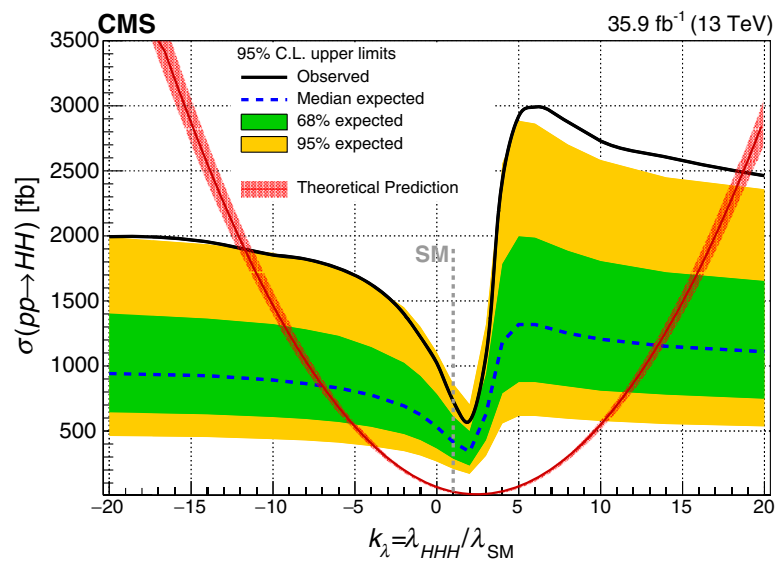

FIG. 2. Expected and observed $95 \%$ C.L. upper limits on the $\mathrm{HH}$ production cross section as a function of the $k_{\lambda}$ parameter. The inner (green) band and the outer (yellow) band indicate the regions containing 68 and $95 \%$, respectively, of the limits on the $\mathrm{HH}$ cross section expected under the background-only hypothesis. The red band shows the expected theoretical [26] cross section expectations and its uncertainty. All other couplings and EFT parameters are set to their SM values. 
emission of an $H H$ pair from a top quark loop. The minimum at $k_{\lambda}=2.46$ corresponds to the maximum negative interference between the two diagrams, which results in a minimum of the cross section but at the same time enhances the relative importance of tails in the $M_{H H}$ distribution. The maximum at $k_{\lambda} \approx 5$ is due to the softness of the $M_{H H}$ spectrum for such values of the trilinear coupling, causing a larger fraction of events to fall outside experimental acceptance. As $\left|k_{\lambda}\right|$ increases, the production via the trilinear Higgs coupling becomes dominant and the limit asymptotically approaches the same value for both $k_{\lambda} \ll-10$ and $k_{\lambda} \gg 10$. This is reflected in the observed exclusion limit as well, where the significance of the small observed excess is relatively less important in the more sensitive small $k_{\lambda}$ region than at large values of $k_{\lambda}$. When fixing all the other EFT parameters to their SM values, the $k_{\lambda}$ parameter is observed (expected) to be constrained to the range $-11.8<k_{\lambda}<18.8\left(-7.1<k_{\lambda}<13.6\right)$ at $95 \%$ C.L. The observed exclusions for the different EFT benchmarks [26] are in the range of 100-3000 $\mathrm{fb}$, and can be seen in Supplemental Material [58]. A small excess, similar to that observed at the SM value, is present across most of the phase space with the exception of the more boosted topologies.

The resonant search is performed in the range of masses from 250 to $3000 \mathrm{GeV}$. Under the hypothesis of a narrowwidth resonance, no significant excess is found across the whole range for either a spin- 0 or a spin- 2 resonance. The results of the combined resonant search are shown in Fig. 3 for the spin-0 model, and in the Supplemental Material [58] for the spin-2 case.

In summary, a combination of searches for nonresonant and resonant Higgs boson pair production has been presented. The combination includes the $b b \gamma \gamma, b b \tau \tau$,

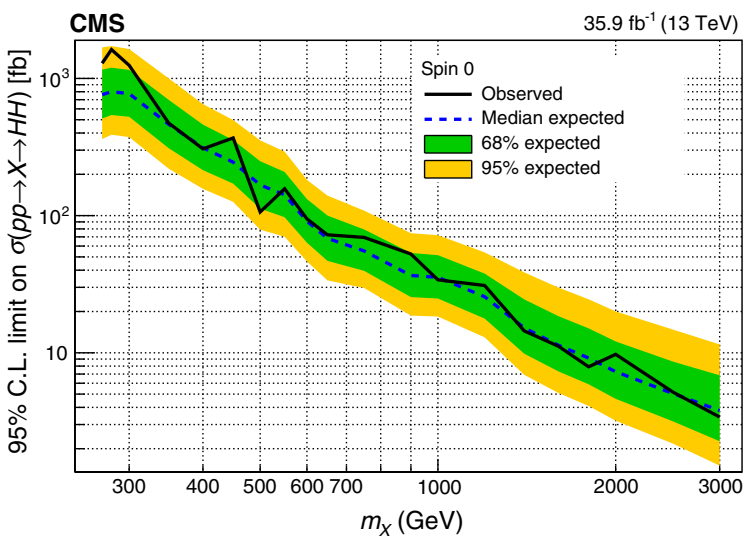

FIG. 3. Expected (dashed) and observed (solid line) 95\% C.L. exclusion limits on the production of a narrow, spin zero resonance $(X)$ decaying into a pair of Higgs bosons. The inner (green) band and the outer (yellow) band indicate the regions containing 68 and 95\%, respectively, of the limits on the $\mathrm{HH}$ cross section expected under the background-only hypothesis. $b b b b$, and $b b V V$ channels, where $V$ represents a $W$ or $Z$ boson, using a data sample collected in proton-proton collisions at $\sqrt{s}=13 \mathrm{TeV}$, which corresponds to an integrated luminosity of $35.9 \mathrm{fb}^{-1}$. Upper limits at 95\% confidence level (C.L.) on the Higgs boson pair production cross section are obtained. For the nonresonant production mechanism, the observed (expected) 95\% C.L. corresponds to 22.2 (12.8) times the theoretical prediction for the standard model cross section. An effective field theory framework is used to parametrize the cross section as a function of anomalous couplings of the Higgs boson. When varying only the ratio $k_{\lambda}$ between the Higgs trilinear coupling $\lambda_{H H H}$ and its standard model expectation, values of $k_{\lambda}$ in the region $-11.8<k_{\lambda}<18.8\left(-7.1<k_{\lambda}<13.6\right)$ are still allowed by the observed (expected) data. For the resonant production mechanism, upper exclusion limits at 95\% C.L. are obtained for the production of a narrow resonance with mass ranging from 250 to $3000 \mathrm{GeV}$. These results represent both the most sensitive and most comprehensive study of double Higgs production at the LHC to date.

We congratulate our colleagues in the CERN accelerator departments for the excellent performance of the LHC and thank the technical and administrative staffs at CERN and at other CMS institutes for their contributions to the success of the CMS effort. In addition, we gratefully acknowledge the computing centers and personnel of the Worldwide LHC Computing Grid for delivering so effectively the computing infrastructure essential to our analyses. Finally, we acknowledge the enduring support for the construction and operation of the LHC and the CMS detector provided by the following funding agencies: BMBWF and FWF (Austria); FNRS and FWO (Belgium); CNPq, CAPES, FAPERJ, FAPERGS, and FAPESP (Brazil); MES (Bulgaria); CERN; CAS, MoST, and NSFC (China); COLCIENCIAS (Colombia); MSES and CSF (Croatia); RPF (Cyprus); SENESCYT (Ecuador); MoER, ERC IUT, and ERDF (Estonia); Academy of Finland, MEC, and HIP (Finland); CEA and CNRS/IN2P3 (France); BMBF, DFG, and HGF (Germany); GSRT (Greece); NKFIA (Hungary); DAE and DST (India); IPM (Iran); SFI (Ireland); INFN (Italy); MSIP and NRF (Republic of Korea); MES (Latvia); LAS (Lithuania); MOE and UM (Malaysia); BUAP, CINVESTAV, CONACYT, LNS, SEP, and UASLP-FAI (Mexico); MOS (Montenegro); MBIE (New Zealand); PAEC (Pakistan); MSHE and NSC (Poland); FCT (Portugal); JINR (Dubna); MON, RosAtom, RAS, RFBR, and NRC KI (Russia); MESTD (Serbia); SEIDI, CPAN, PCTI, and FEDER (Spain); MOSTR (Sri Lanka); Swiss Funding Agencies (Switzerland); MST (Taipei); ThEPCenter, IPST, STAR, and NSTDA (Thailand); TUBITAK and TAEK (Turkey); NASU and SFFR (Ukraine); STFC (United Kingdom); DOE and NSF (USA). 
[1] ATLAS Collaboration, Observation of a new particle in the search for the standard model Higgs boson with the ATLAS detector at the LHC, Phys. Lett. B 716, 1 (2012).

[2] CMS Collaboration, Observation of a new boson at a mass of $125 \mathrm{GeV}$ with the CMS experiment at the LHC, Phys. Lett. B 716, 30 (2012).

[3] CMS Collaboration, Observation of a new boson with mass near $125 \mathrm{GeV}$ in pp collisions at $\sqrt{s}=7$ and $8 \mathrm{TeV}$, J. High Energy Phys. 06 (2013) 081.

[4] ATLAS and CMS Collaborations, Combined Measurement of the Higgs Boson Mass in $p p$ Collisions at $\sqrt{s}=7$ and $8 \mathrm{TeV}$ with the ATLAS and CMS Experiments, Phys. Rev. Lett. 114, 191803 (2015).

[5] CMS Collaboration, Measurements of properties of the Higgs boson decaying into the four-lepton final state in pp collisions at $\sqrt{s}=13 \mathrm{TeV}$, J. High Energy Phys. 11 (2017) 047.

[6] ATLAS and CMS Collaborations, Measurements of the Higgs boson production and decay rates and constraints on its couplings from a combined ATLAS and CMS analysis of the LHC pp collision data at $\sqrt{s}=7$ and $8 \mathrm{TeV}$, J. High Energy Phys. 08 (2016) 045.

[7] CMS Collaboration, Combined measurements of Higgs boson couplings in proton-proton collisions at $\sqrt{s}=13 \mathrm{TeV}$, arXiv: 1809.10733.

[8] J. Baglio, A. Djouadi, R. Gröber, M. M. Mühlleitner, J. Quevillon, and M. Spira, The measurement of the Higgs self-coupling at the LHC: Theoretical status, J. High Energy Phys. 04 (2013) 151.

[9] T. Binoth and J. J. van der Bij, Influence of strongly coupled, hidden scalars on Higgs signals, Z. Phys. C 75, 17 (1997).

[10] R. M. Schabinger and J. D. Wells, Minimal spontaneously broken hidden sector and its impact on Higgs boson physics at the CERN large hadron collider, Phys. Rev. D 72, 093007 (2005).

[11] B. Patt and F. Wilczek, Higgs-field portal into hidden sectors, arXiv:hep-ph/0605188.

[12] G. C. Branco, P. M. Ferreira, L. Lavoura, M. N. Rebelo, M. Sher, and J. P. Silva, Theory and phenomenology of twoHiggs-doublet models, Phys. Rep. 516, 1 (2012).

[13] P. Fayet, Supergauge invariant extension of the Higgs mechanism and a model for the electron and its neutrino, Nucl. Phys. B90, 104 (1975).

[14] P. Fayet, Spontaneously broken supersymmetric theories of weak, electromagnetic and strong interactions, Phys. Lett. 69B, 489 (1977).

[15] K. Agashe, H. Davoudiasl, G. Perez, and A. Soni, Warped gravitons at the CERN LHC and beyond, Phys. Rev. D 76, 036006 (2007).

[16] A. L. Fitzpatrick, J. Kaplan, L. Randall, and L.-T. Wang, Searching for the Kaluza-Klein graviton in bulk RS models, J. High Energy Phys. 09 (2007) 013.

[17] LHC Higgs Cross Section Working Group, Handbook of LHC Higgs Cross Sections: 4. Deciphering the Nature of the Higgs Sector (CERN, Geneva, 2016).

[18] S. Borowka, N. Greiner, G. Heinrich, S. P. Jones, M. Kerner, J. Schlenk, U. Schubert, and T. Zirke, Higgs Boson Pair Production in Gluon Fusion at Next-to-Leading Order with
Full Top-Quark Mass Dependence, Phys. Rev. Lett. 117, 012001 (2016); 117, 079901(E) (2016).

[19] S. Borowka, N. Greiner, G. Heinrich, S. P. Jones, M. Kerner, J. Schlenk, and T. Zirke, Full top quark mass dependence in Higgs boson pair production at NLO, J. High Energy Phys. 10 (2016) 107.

[20] D. de Florian and J. Mazzitelli, Higgs pair production at next-to-next-to-leading logarithmic accuracy at the LHC, J. High Energy Phys. 09 (2015) 053.

[21] G. Degrassi, P. Paolo Giardino, and R. Grober, On the twoloop virtual QCD corrections to Higgs boson pair production in the standard model, Eur. Phys. J. C 76, 411 (2016).

[22] M. Botje, J. Butterworth, A. Cooper-Sarkar, A. De Roeck, J. Feltesse, S. Forte, A. Glazov, J. JHuston, R. McNulty, T. Sjostrand, and R. Thorne, The PDF4LHC Working Group Interim Recommendations, arXiv:1101.0538.

[23] S. Alekhin et al., The PDF4LHC working group interim report, arXiv:1101.0536.

[24] R. D. Ball et al. (NNPDF Collaboration), Parton distributions for the LHC Run II, J. High Energy Phys. 04 (2015) 040.

[25] F. Goertz, A. Papaefstathiou, L. L. Yang, and J. Zurita, Higgs boson pair production in the $d=6$ extension of the SM, J. High Energy Phys. 04 (2015) 167.

[26] A. Carvalho, M. Dall'Osso, P. De Castro Manzano, T. Dorigo, F. Goertz, M. Gouzevich, and M. Tosi, Analytical parametrization and shape classification of anomalous $\mathrm{HH}$ production in the EFT approach, arXiv:1608.06578.

[27] ATLAS Collaboration, Searches for Higgs boson pair production in the $h h \rightarrow b b \tau \tau, \gamma \gamma W W^{*}, \gamma \gamma b b, b b b b$ channels with the ATLAS detector, Phys. Rev. D 92, 092004 (2015).

[28] CMS Collaboration, Search for two Higgs bosons in final states containing two photons and two bottom quarks in proton-proton collisions at $8 \mathrm{TeV}$, Phys. Rev. D 94, 052012 (2016).

[29] CMS Collaboration, Search for Higgs boson pair production in the $b b \tau \tau$ final state in proton-proton collisions at $\sqrt{s}=8$ TeV, Phys. Rev. D 96, 072004 (2017).

[30] CMS Collaboration, Search for Higgs boson pair production in the $\gamma \gamma \mathrm{bb}$ final state in pp collisions at $\sqrt{s}=13 \mathrm{TeV}$, Phys. Lett. B 788, 7 (2019).

[31] ATLAS Collaboration, Search for Higgs boson pair production in the $\gamma \gamma b \bar{b}$ final state with $13 \mathrm{TeV} p p$ collision data collected by the ATLAS experiment, J. High Energy Phys. 11 (2018) 040.

[32] CMS Collaboration, Search for Higgs boson pair production in events with two bottom quarks and two tau leptons in proton-proton collisions at $\sqrt{s}=13 \mathrm{TeV}$, Phys. Lett. B 778, 101 (2018).

[33] ATLAS Collaboration, A Search for Resonant and NonResonant Higgs Boson Pair Production in the $b \bar{b} \tau^{+} \tau^{-}$ Decay Channel in $p p$ Collisions at $\sqrt{s}=13 \mathrm{TeV}$ with the ATLAS Detector, Phys. Rev. Lett. 121, 191801 (2018).

[34] CMS Collaboration, Search for resonant pair production of Higgs bosons decaying to bottom quark-antiquark pairs in proton-proton collisions at $13 \mathrm{TeV}$, J. High Energy Phys. 08 (2018) 152.

[35] CMS Collaboration, Search for a massive resonance decaying to a pair of Higgs bosons in the four $b$ quark final state in proton-proton collisions at $\sqrt{s}=13 \mathrm{TeV}$, Phys. Lett. B 781, 244 (2018). 
[36] CMS Collaboration, Search for production of Higgs boson pairs in the four $\mathrm{b}$ quark final state using large-area jets in proton-proton collisions at $\sqrt{s}=13 \mathrm{TeV}$, J. High Energy Phys. 01 (2019) 040.

[37] CMS Collaboration, Search for nonresonant Higgs boson pair production in the $b \bar{b} b \bar{b}$ final state at $\sqrt{s}=13 \mathrm{TeV}$, arXiv:1810.11854.

[38] ATLAS Collaboration, Search for pair production of Higgs bosons in the $b \bar{b} b \bar{b}$ final state using proton-proton collisions at $\sqrt{s}=13 \mathrm{TeV}$ with the ATLAS detector, J. High Energy Phys. 01 (2019) 030.

[39] CMS Collaboration, Search for resonant and nonresonant Higgs boson pair production in the $\mathrm{b} \overline{\mathrm{b}} \ell \nu \ell \nu$ final state in proton-proton collisions at $\sqrt{s}=13 \mathrm{TeV}$, J. High Energy Phys. 01 (2018) 054.

[40] CMS Collaboration, The CMS trigger system, J. Instrum. 12, P01020 (2017).

[41] CMS Collaboration, Particle-flow reconstruction and global event description with the CMS detector, J. Instrum. 12, P10003 (2017).

[42] CMS Collaboration, Identification of heavy-flavour jets with the CMS detector in pp collisions at $13 \mathrm{TeV}$, J. Instrum. 13, P05011 (2018).

[43] CMS Collaboration, The CMS experiment at the CERN LHC, J. Instrum. 3, S08004 (2008).

[44] L. Bianchini, J. Conway, E. K. Friis, and C. Veelken, Reconstruction of the Higgs mass in $H \rightarrow \tau \tau$ events by dynamical likelihood techniques, J. Phys. Conf. Ser. 513, 022035 (2014).

[45] A. J. Barr, M. J. Dolan, C. Englert, and M. Spannowsky, Di-Higgs final states augMT2ed-selecting $h h$ events at the high luminosity LHC, Phys. Lett. B 728, 308 (2014).

[46] C. G. Lester and D. J. Summers, Measuring masses of semiinvisibly decaying particle pairs produced at hadron colliders, Phys. Lett. B 463, 99 (1999).

[47] CMS Collaboration, Searches for a heavy scalar boson $\mathrm{H}$ decaying to a pair of $125 \mathrm{GeV}$ Higgs bosons hh or for a heavy pseudoscalar boson A decaying to $\mathrm{Zh}$, in the final states with $h \rightarrow \tau \tau$, Phys. Lett. B 755, 217 (2016).

[48] J. M. Butterworth, A. R. Davison, M. Rubin, and G. P. Salam, Jet Substructure as a New Higgs Search Channel at the LHC, Phys. Rev. Lett. 100, 242001 (2008).
[49] W. Verkerke and D. P. Kirkby, The RooFit toolkit for data modeling, in Proceedings of the 13th International Conference for Computing in High-Energy and Nuclear Physics (CHEP03) (2003), [eConf C0303241, MOLT007], http://inspirehep.net/record/634021.

[50] L. Moneta, K. Belasco, K. S. Cranmer, S. Kreiss, A. Lazzaro, D. Piparo, G. Schott, W. Verkerke, and M. Wolf, The RooStats project, in Proceedings of the 13th International Workshop on Advanced computing and analysis techniques in physics research (ACAT2010) (Jaipur, India, 2010), p. 057; Proc. Sci. ACAT2010 (093) 057.

[51] ATLAS and CMS Collaborations, The LHC Higgs Combination Group, Procedure for the LHC Higgs boson search combination in Summer 2011, Technical Report No. CMSNOTE-2011-005, and Report No. ATL-PHYS-PUB-201111, 2011.

[52] G. Cowan, K. Cranmer, E. Gross, and O. Vitells, Asymptotic formulae for likelihood-based tests of new physics, Eur. Phys. J. C 71, 1554 (2011); Erratum, Eur. Phys. J. C 73, 2501(E) (2013).

[53] T. R. Junk, A. Korytov, and A. L. Read, Appendix: Statistical methods, in Discovery of the Higgs Boson, edited by A. Nisati and V. Sharma (World Scientific, Singapore, 2016), p. 415.

[54] A. L. Read, Presentation of search results: The $\mathrm{CL}_{\mathrm{s}}$ technique, J. Phys. G 28, 2693 (2002).

[55] T. Junk, Confidence level computation for combining searches with small statistics, Nucl. Instrum. Methods Phys. Res., Sect. A 434, 435 (1999).

[56] CMS Collaboration, CMS Luminosity Measurements for the 2016 Data Taking Period, Technical Report No. CMSPAS-LUM-17-001, 2017, https://cds.cern.ch/record/ 2257069.

[57] R. Frederix, S. Frixione, V. Hirschi, F. Maltoni, O. Mattelaer, P. Torrielli, E. Vryonidou, and M. Zaro, Higgs pair production at the LHC with NLO and parton-shower effects, Phys. Lett. B 732, 142 (2014).

[58] See Supplemental Material at http://link.aps.org/ supplemental/10.1103/PhysRevLett.122.121803 for the results of the benchmark shape studies results and for the resonant exclusion limit under the spin-2 assumption.

A. M. Sirunyan, ${ }^{1}$ A. Tumasyan, ${ }^{1}$ W. Adam, ${ }^{2}$ F. Ambrogi, ${ }^{2}$ E. Asilar, ${ }^{2}$ T. Bergauer, ${ }^{2}$ J. Brandstetter, ${ }^{2}$ M. Dragicevic, ${ }^{2}$ J. Erö, ${ }^{2}$ A. Escalante Del Valle, ${ }^{2}$ M. Flechl, ${ }^{2}$ R. Frühwirth, ${ }^{2, b}$ V. M. Ghete, ${ }^{2}$ J. Hrubec, ${ }^{2}$ M. Jeitler, ${ }^{2, b}$ N. Krammer, ${ }^{2}$ I. Krätschmer, ${ }^{2}$ D. Liko, ${ }^{2}$ T. Madlener, ${ }^{2}$ I. Mikulec, ${ }^{2}$ N. Rad, ${ }^{2}$ H. Rohringer, ${ }^{2}$ J. Schieck, ${ }^{2, b}$ R. Schöfbeck, ${ }^{2}$ M. Spanring, ${ }^{2}$ D. Spitzbart, ${ }^{2}$ A. Taurok, ${ }^{2}$ W. Waltenberger, ${ }^{2}$ J. Wittmann, ${ }^{2}$ C.-E. Wulz, ${ }^{2, b}$ M. Zarucki, ${ }^{2}$ V. Chekhovsky, ${ }^{3}$ V. Mossolov, ${ }^{3}$ J. Suarez Gonzalez, ${ }^{3}$ E. A. De Wolf, ${ }^{4}$ D. Di Croce, ${ }^{4}$ X. Janssen, ${ }^{4}$ J. Lauwers, ${ }^{4}$ M. Pieters, ${ }^{4}$ H. Van Haevermaet, ${ }^{4}$ P. Van Mechelen, ${ }^{4}$ N. Van Remortel, ${ }^{4}$ S. Abu Zeid, ${ }^{5}$ F. Blekman, ${ }^{5}$ J. D'Hondt, ${ }^{5}$ J. De Clercq, ${ }^{5}$ K. Deroover, ${ }^{5}$ G. Flouris, D. Lontkovskyi, ${ }^{5}$ S. Lowette, ${ }^{5}$ I. Marchesini ${ }^{5}$ S. Moortgat,${ }^{5}$ L. Moreels, ${ }^{5}$ Q. Python, ${ }^{5}$ K. Skovpen, ${ }^{5}$ S. Tavernier, ${ }^{5}$ W. Van Doninck, ${ }^{5}$ P. Van Mulders, ${ }^{5}$ I. Van Parijs, ${ }^{5}$ D. Beghin, ${ }^{6}$ B. Bilin, ${ }^{6}$ H. Brun, ${ }^{6}$ B. Clerbaux, ${ }^{6}$ G. De Lentdecker, ${ }^{6}$ H. Delannoy, ${ }^{6}$ B. Dorney, ${ }^{6}$ G. Fasanella, ${ }^{6}$ L. Favart ${ }^{6}$ R. Goldouzian, ${ }^{6}$ A. Grebenyuk, ${ }^{6}$ A. K. Kalsi, ${ }^{6}$ T. Lenzi, ${ }^{6}$ J. Luetic, ${ }^{6}$ N. Postiau, ${ }^{6}$ E. Starling, ${ }^{6}$ L. Thomas, ${ }^{6}$ C. Vander Velde, ${ }^{6}$ P. Vanlaer, ${ }^{6}$ D. Vannerom, ${ }^{6}$ Q. Wang, ${ }^{6}$ T. Cornelis, ${ }^{7}$ D. Dobur, ${ }^{7}$ A. Fagot, ${ }^{7}$ M. Gul, ${ }^{7}$ I. Khvastunov, ${ }^{7, c}$ D. Poyraz, ${ }^{7}$ C. Roskas, ${ }^{7}$ D. Trocino, ${ }^{7}$ M. Tytgat, ${ }^{7}$ W. Verbeke, ${ }^{7}$ B. Vermassen, ${ }^{7}$ M. Vit, ${ }^{7}$ 
N. Zaganidis, ${ }^{7}$ H. Bakhshiansohi, ${ }^{8}$ O. Bondu, ${ }^{8}$ S. Brochet, ${ }^{8}$ G. Bruno, ${ }^{8}$ C. Caputo, ${ }^{8}$ P. David, ${ }^{8}$ C. Delaere, ${ }^{8}$ M. Delcourt, ${ }^{8}$ A. Giammanco, ${ }^{8}$ G. Krintiras, ${ }^{8}$ V. Lemaitre, ${ }^{8}$ A. Magitteri, ${ }^{8}$ K. Piotrzkowski, ${ }^{8}$ A. Saggio, ${ }^{8}$ M. Vidal Marono, ${ }^{8}$ S. Wertz, ${ }^{8}$ J. Zobec ${ }^{8}$ F. L. Alves, ${ }^{9}$ G. A. Alves, ${ }^{9}$ M. Correa Martins Junior, ${ }^{9}$ G. Correia Silva, ${ }^{9}$ C. Hensel,,${ }^{9}$ A. Moraes, ${ }^{9}$ M. E. Pol, ${ }^{9}$ P. Rebello Teles, ${ }^{9}$ E. Belchior Batista Das Chagas, ${ }^{10}$ W. Carvalho, ${ }^{10}$ J. Chinellato, ${ }^{10, d}$ E. Coelho, ${ }^{10}$ E. M. Da Costa, ${ }^{10}$ G. G. Da Silveira, ${ }^{10, e}$ D. De Jesus Damiao, ${ }^{10}$ C. De Oliveira Martins, ${ }^{10}$ S. Fonseca De Souza,${ }^{10}$ H. Malbouisson,,${ }^{10}$ D. Matos Figueiredo, ${ }^{10}$ M. Melo De Almeida, ${ }^{10}$ C. Mora Herrera, ${ }^{10}$ L. Mundim,${ }^{10}$ H. Nogima,${ }^{10}$ W. L. Prado Da Silva, ${ }^{10}$ L. J. Sanchez Rosas, ${ }^{10}$ A. Santoro ${ }^{10}$ A. Sznajder, ${ }^{10}$ M. Thiel,${ }^{10}$ E. J. Tonelli Manganote, ${ }^{10, d}$ F. Torres Da Silva De Araujo, ${ }^{10}$ A. Vilela Pereira, ${ }^{10}$ S. Ahuja, ${ }^{11 \mathrm{a}}$ C. A. Bernardes, ${ }^{11 \mathrm{a}}$ L. Calligaris, ${ }^{11 \mathrm{a}}$ T. R. Fernandez Perez Tomei, ${ }^{11 \mathrm{a}}$ E. M. Gregores, ${ }^{11 \mathrm{a}, 11 \mathrm{~b}}$ P. G. Mercadante, ${ }^{11 \mathrm{a}, 11 \mathrm{~b}}$ S. F. Novaes, ${ }^{11 \mathrm{a}}$ Sandra S. Padula, ${ }^{11 \mathrm{a}}$ A. Aleksandrov, ${ }^{12}$ R. Hadjiiska, ${ }^{12}$ P. Iaydjiev, ${ }^{12}$ A. Marinov, ${ }^{12}$ M. Misheva,${ }^{12}$ M. Rodozov, ${ }^{12}$ M. Shopova, ${ }^{12}$ G. Sultanov, ${ }^{12}$ A. Dimitrov, ${ }^{13}$ L. Litov, ${ }^{13}$ B. Pavlov, ${ }^{13}$ P. Petkov, ${ }^{13}$ W. Fang,,${ }^{14, f}$ X. Gao, ${ }^{14, \mathrm{f}}$ L. Yuan, ${ }^{14}$ M. Ahmad, ${ }^{15}$ J. G. Bian, ${ }^{15}$ G. M. Chen, ${ }^{15}$ H. S. Chen, ${ }^{15}$ M. Chen, ${ }^{15}$ Y. Chen, ${ }^{15}$ C. H. Jiang, ${ }^{15}$ D. Leggat, ${ }^{15}$ H. Liao, ${ }^{15}$ Z. Liu, ${ }^{15}$ F. Romeo,${ }^{15}$ S. M. Shaheen, ${ }^{15, g}$ A. Spiezia, ${ }^{15}$ J. Tao,${ }^{15}$ Z. Wang,${ }^{15}$ E. Yazgan, ${ }^{15}$ H. Zhang,${ }^{15}$ S. Zhang, ${ }^{15,9}$ J. Zhao, ${ }^{15}$ Y. Ban, ${ }^{16}$ G. Chen, ${ }^{16}$ A. Levin, ${ }^{16}$ J. Li,${ }^{16}$ L. Li, ${ }^{16}$ Q. Li, ${ }^{16}$ Y. Mao, ${ }^{16}$ S. J. Qian, ${ }^{16}$ D. Wang, ${ }^{16}$ Y. Wang, ${ }^{17}$ C. Avila, ${ }^{18}$ A. Cabrera, ${ }^{18}$ C. A. Carrillo Montoya, ${ }^{18}$ L. F. Chaparro Sierra ${ }^{18}$ C. Florez, ${ }^{18}$

C. F. González Hernández, ${ }^{18}$ M. A. Segura Delgado, ${ }^{18}$ B. Courbon, ${ }^{19}$ N. Godinovic, ${ }^{19}$ D. Lelas, ${ }^{19}$ I. Puljak, ${ }^{19}$ T. Sculac, ${ }^{19}$ Z. Antunovic, ${ }^{20}$ M. Kovac, ${ }^{20}$ V. Brigljevic, ${ }^{21}$ D. Ferencek ${ }^{21}$ K. Kadija, ${ }^{21}$ B. Mesic, ${ }^{21}$ A. Starodumov, ${ }^{21, h}$ T. Susa, ${ }^{21}$ M. W. Ather ${ }^{22}$ A. Attikis, ${ }^{22}$ M. Kolosova, ${ }^{22}$ G. Mavromanolakis, ${ }^{22}$ J. Mousa, ${ }^{22}$ C. Nicolaou, ${ }^{22}$ F. Ptochos, ${ }^{22}$ P. A. Razis, ${ }^{22}$ H. Rykaczewski, ${ }^{22}$ M. Finger, ${ }^{23, i}$ M. Finger Jr., ${ }^{23, i}$ E. Ayala, ${ }^{24}$ E. Carrera Jarrin, ${ }^{25}$ H. Abdalla, ${ }^{26, j}$ A. A. Abdelalim, ${ }^{26, k, 1}$ A. Mohamed, ${ }^{26,1}$ S. Bhowmik, ${ }^{27}$ A. Carvalho Antunes De Oliveira, ${ }^{27}$ R. K. Dewanjee, ${ }^{27}$ K. Ehataht, ${ }^{27}$ M. Kadastik, ${ }^{27}$ M. Raidal, ${ }^{27}$ C. Veelken, ${ }^{27}$ P. Eerola,${ }^{28}$ H. Kirschenmann, ${ }^{28}$ J. Pekkanen, ${ }^{28}$ M. Voutilainen, ${ }^{28}$ J. Havukainen, ${ }^{29}$

J. K. Heikkilä, ${ }^{29}$ T. Järvinen, ${ }^{29}$ V. Karimäki, ${ }^{29}$ R. Kinnunen, ${ }^{29}$ T. Lampén, ${ }^{29}$ K. Lassila-Perini, ${ }^{29}$ S. Laurila, ${ }^{29}$ S. Lehti, ${ }^{29}$ T. Lindén, ${ }^{29}$ P. Luukka, ${ }^{29}$ T. Mäenpää, ${ }^{29}$ H. Siikonen, ${ }^{29}$ E. Tuominen, ${ }^{29}$ J. Tuominiemi, ${ }^{29}$ T. Tuuva, ${ }^{30}$ M. Besancon, ${ }^{31}$ F. Couderc ${ }^{31}$ M. Dejardin, ${ }^{31}$ D. Denegri, ${ }^{31}$ J. L. Faure, ${ }^{31}$ F. Ferri, ${ }^{31}$ S. Ganjour, ${ }^{31}$ A. Givernaud, ${ }^{31}$ P. Gras, ${ }^{31}$ G. Hamel de Monchenault, ${ }^{31}$ P. Jarry, ${ }^{31}$ C. Leloup ${ }^{31}$ E. Locci, ${ }^{31}$ J. Malcles,${ }^{31}$ G. Negro, ${ }^{31}$ J. Rander, ${ }^{31}$ A. Rosowsky, ${ }^{31}$ M. Ö. Sahin, ${ }^{31}$ M. Titov,${ }^{31}$ A. Abdulsalam, ${ }^{32, \mathrm{~m}}$ C. Amendola,${ }^{32}$ I. Antropov, ${ }^{32}$ F. Beaudette, ${ }^{32}$ P. Busson,${ }^{32}$ C. Charlot, ${ }^{32}$ R. Granier de Cassagnac, ${ }^{32}$ I. Kucher, ${ }^{32}$ A. Lobanov, ${ }^{32}$ J. Martin Blanco, ${ }^{32}$ C. Martin Perez, ${ }^{32}$ M. Nguyen, ${ }^{32}$ C. Ochando, ${ }^{32}$ G. Ortona, ${ }^{32}$ P. Paganini, ${ }^{32}$ P. Pigard ${ }^{32}$ J. Rembser, ${ }^{32}$ R. Salerno, ${ }^{32}$ J. B. Sauvan, ${ }^{32}$ Y. Sirois, ${ }^{32}$ A. G. Stahl Leiton, ${ }^{32}$ A. Zabi, ${ }^{32}$ A. Zghiche, ${ }^{32}$ J.-L. Agram, ${ }^{33, n}$ J. Andrea, ${ }^{33}$ D. Bloch,${ }^{33}$ J.-M. Brom, ${ }^{33}$ E. C. Chabert, ${ }^{33}$ V. Cherepanov, ${ }^{33}$ C. Collard, ${ }^{33}$ E. Conte, ${ }^{33, n}$ J.-C. Fontaine, ${ }^{33, n}$ D. Gelé, ${ }^{33}$ U. Goerlach, ${ }^{33}$ M. Jansová, ${ }^{33}$ A.-C. Le Bihan, ${ }^{33}$ N. Tonon, ${ }^{33}$ P. Van Hove, ${ }^{33}$ S. Gadrat, ${ }^{34}$ S. Beauceron, ${ }^{35}$ C. Bernet, ${ }^{35}$ G. Boudoul, ${ }^{35}$ N. Chanon, ${ }^{35}$ R. Chierici, ${ }^{35}$ D. Contardo, ${ }^{35}$ P. Depasse, ${ }^{35}$ H. El Mamouni, ${ }^{35}$ J. Fay, ${ }^{35}$ L. Finco, ${ }^{35}$ S. Gascon,${ }^{35}$ M. Gouzevitch, ${ }^{35}$ G. Grenier, ${ }^{35}$ B. Ille,${ }^{35}$ F. Lagarde,${ }^{35}$ I. B. Laktineh, ${ }^{35}$ H. Lattaud, ${ }^{35}$ M. Lethuillier, ${ }^{35}$ L. Mirabito, ${ }^{35}$ S. Perries, ${ }^{35}$ A. Popov,${ }^{35,0}$ V. Sordini, ${ }^{35}$ G. Touquet, ${ }^{35}$ M. Vander Donckt, ${ }^{35}$ S. Viret, ${ }^{35}$ A. Khvedelidze, ${ }^{36, i}$ Z. Tsamalaidze, ${ }^{37, i}$ C. Autermann, ${ }^{38}$ L. Feld, ${ }^{38}$ M. K. Kiesel, ${ }^{38}$ K. Klein, ${ }^{38}$ M. Lipinski, ${ }^{38}$ M. Preuten, ${ }^{38}$ M. P. Rauch, ${ }^{38}$ C. Schomakers, ${ }^{38}$ J. Schulz,${ }^{38}$ M. Teroerde, ${ }^{38}$ B. Wittmer, ${ }^{38}$ A. Albert, ${ }^{39}$ D. Duchardt, ${ }^{39}$ M. Erdmann, ${ }^{39}$ S. Erdweg, ${ }^{39}$ T. Esch, ${ }^{39}$ R. Fischer, ${ }^{39}$ S. Ghosh ${ }^{39}$ A. Güth, ${ }^{39}$ T. Hebbeker, ${ }^{39}$ C. Heidemann, ${ }^{39}$ K. Hoepfner, ${ }^{39}$ H. Keller, ${ }^{39}$ L. Mastrolorenzo, ${ }^{39}$ M. Merschmeyer, ${ }^{39}$ A. Meyer, ${ }^{39}$ P. Millet, ${ }^{39}$ S. Mukherjee, ${ }^{39}$ T. Pook,${ }^{39}$ M. Radziej,${ }^{39}$ H. Reithler, ${ }^{39}$ M. Rieger, ${ }^{39}$ A. Schmidt,${ }^{39}$ D. Teyssier, ${ }^{39}$ S. Thüer, ${ }^{39}$ G. Flügge, ${ }^{40}$ O. Hlushchenko, ${ }^{40}$ T. Kress, ${ }^{40}$ T. Müller, ${ }^{40}$ A. Nehrkorn, ${ }^{40}$ A. Nowack, ${ }^{40}$ C. Pistone, ${ }^{40}$ O. Pooth, ${ }^{40}$ D. Roy, ${ }^{40}$ H. Sert, ${ }^{40}$ A. Stahl,${ }^{40, p}$ M. Aldaya Martin, ${ }^{41}$ T. Arndt ${ }^{41}$ C. Asawatangtrakuldee, ${ }^{41}$ I. Babounikau, ${ }^{41}$ K. Beernaert ${ }^{41}$ O. Behnke, ${ }^{41}$ U. Behrens, ${ }^{41}$ A. Bermúdez Martínez, ${ }^{41}$ D. Bertsche, ${ }^{41}$ A. A. Bin Anuar, ${ }^{41}$ K. Borras, ${ }^{41, q}$ V. Botta ${ }^{41}$ A. Campbell, ${ }^{41}$ P. Connor, ${ }^{41}$ C. Contreras-Campana, ${ }^{41}$ V. Danilov, ${ }^{41}$ A. De Wit, ${ }^{41}$ M. M. Defranchis, ${ }^{41}$ C. Diez Pardos, ${ }^{41}$ D. Domínguez Damiani, ${ }^{41}$ G. Eckerlin, ${ }^{41}$ T. Eichhorn, ${ }^{41}$ A. Elwood, ${ }^{41}$ E. Eren, ${ }^{41}$ E. Gallo, ${ }^{41, r}$ A. Geiser,${ }^{41}$ J. M. Grados Luyando, ${ }^{41}$ A. Grohsjean, ${ }^{41}$ M. Guthoff, ${ }^{41}$ M. Haranko, ${ }^{41}$ A. Harb,${ }^{41}$ J. Hauk, ${ }^{41}$ H. Jung, ${ }^{41}$ M. Kasemann, ${ }^{41}$ J. Keaveney, ${ }^{41}$ C. Kleinwort, ${ }^{41}$ J. Knolle, ${ }^{41}$ D. Krücker, ${ }^{41}$ W. Lange,${ }^{41}$ A. Lelek ${ }^{41}$ T. Lenz, ${ }^{41}$ J. Leonard ${ }^{41}$ K. Lipka, ${ }^{41}$ W. Lohmann, ${ }^{41, \mathrm{~s}}$ R. Mankel, ${ }^{41}$ I.-A. Melzer-Pellmann, ${ }^{41}$ A. B. Meyer, ${ }^{41}$ M. Meyer,${ }^{41}$ M. Missiroli, ${ }^{41}$ G. Mittag, ${ }^{41}$ J. Mnich, ${ }^{41}$ V. Myronenko, ${ }^{41}$ S. K. Pflitsch,${ }^{41}$ D. Pitzl, ${ }^{41}$ A. Raspereza, ${ }^{41}$ M. Savitskyi,${ }^{41}$ P. Saxena,${ }^{41}$ P. Schütze,${ }^{41}$ C. Schwanenberger,${ }^{41}$ R. Shevchenko, ${ }^{41}$ A. Singh ${ }^{41}$ H. Tholen, ${ }^{41}$ O. Turkot, ${ }^{41}$ A. Vagnerini, ${ }^{41}$ G. P. Van Onsem, ${ }^{41}$ R. Walsh,${ }^{41}$ Y. Wen, ${ }^{41}$ K. Wichmann, ${ }^{41}$ C. Wissing, ${ }^{41}$ O. Zenaiev, ${ }^{41}$ R. Aggleton, ${ }^{42}$ S. Bein, ${ }^{42}$ L. Benato ${ }^{42}$ A. Benecke, ${ }^{42}$ V. Blobel, ${ }^{42}$ T. Dreyer,${ }^{42}$ A. Ebrahimi, ${ }^{42}$ E. Garutti, ${ }^{42}$ D. Gonzalez, ${ }^{42}$ P. Gunnellini, ${ }^{42}$ J. Haller, ${ }^{42}$ A. Hinzmann, ${ }^{42}$ A. Karavdina, ${ }^{42}$ G. Kasieczka, ${ }^{42}$ 
R. Klanner, ${ }^{42}$ R. Kogler, ${ }^{42}$ N. Kovalchuk,${ }^{42}$ S. Kurz ${ }^{42}$ V. Kutzner, ${ }^{42}$ J. Lange, ${ }^{42}$ D. Marconi, ${ }^{42}$ J. Multhaup,${ }^{42}$ M. Niedziela ${ }^{42}$ C. E. N. Niemeyer, ${ }^{42}$ D. Nowatschin, ${ }^{42}$ A. Perieanu, ${ }^{42}$ A. Reimers,${ }^{42}$ O. Rieger, ${ }^{42}$ C. Scharf, ${ }^{42}$ P. Schleper, ${ }^{42}$ S. Schumann ${ }^{42}$ J. Schwandt, ${ }^{42}$ J. Sonneveld, ${ }^{42}$ H. Stadie, ${ }^{42}$ G. Steinbrück, ${ }^{42}$ F. M. Stober, ${ }^{42}$ M. Stöver, ${ }^{42}$ A. Vanhoefer,${ }^{42}$ B. Vormwald, ${ }^{42}$ I. Zoi, ${ }^{42}$ M. Akbiyik, ${ }^{43}$ C. Barth, ${ }^{43}$ M. Baselga ${ }^{43}$ S. Baur, ${ }^{43}$ E. Butz, ${ }^{43}$ R. Caspart, ${ }^{43}$ T. Chwalek, ${ }^{43}$ F. Colombo, ${ }^{43}$ W. De Boer, ${ }^{43}$ A. Dierlamm, ${ }^{43}$ K. El Morabit ${ }^{43}$ N. Faltermann, ${ }^{43}$ B. Freund, ${ }^{43}$ M. Giffels, ${ }^{43}$ M. A. Harrendorf,${ }^{43}$ F. Hartmann, ${ }^{43, p}$ S. M. Heindl, ${ }^{43}$ U. Husemann, ${ }^{43}$ I. Katkov, ${ }^{43,0}$ S. Kudella, ${ }^{43}$ S. Mitra, ${ }^{43}$ M. U. Mozer, ${ }^{43}$ Th. Müller, ${ }^{43}$ M. Musich ${ }^{43}$ M. Plagge, ${ }^{43}$ G. Quast ${ }^{43}$ K. Rabbertz, ${ }^{43}$ M. Schröder,${ }^{43}$ I. Shvetsov,${ }^{43}$ H. J. Simonis, ${ }^{43}$ R. Ulrich,${ }^{43}$ S. Wayand, ${ }^{43}$ M. Weber, ${ }^{43}$ T. Weiler, ${ }^{43}$ C. Wöhrmann, ${ }^{43}$ R. Wolf,${ }^{43}$ G. Anagnostou, ${ }^{44}$ G. Daskalakis, ${ }^{44}$ T. Geralis, ${ }^{44}$ A. Kyriakis, ${ }^{44}$ D. Loukas, ${ }^{44}$ G. Paspalaki, ${ }^{44}$ G. Karathanasis, ${ }^{45}$ P. Kontaxakis, ${ }^{45}$ A. Panagiotou, ${ }^{45}$ I. Papavergou, ${ }^{45}$ N. Saoulidou, ${ }^{45}$ E. Tziaferi, ${ }^{45}$ K. Vellidis, ${ }^{45}$ K. Kousouris ${ }^{46}$ I. Papakrivopoulos, ${ }^{46}$ G. Tsipolitis, ${ }^{46}$ I. Evangelou, ${ }^{47}$ C. Foudas, ${ }^{47}$ P. Gianneios, ${ }^{47}$ P. Katsoulis, ${ }^{47}$ P. Kokkas, ${ }^{47}$ S. Mallios, ${ }^{47}$ N. Manthos, ${ }^{47}$ I. Papadopoulos,${ }^{47}$ E. Paradas, ${ }^{47}$ J. Strologas, ${ }^{47}$ F. A. Triantis, ${ }^{47}$ D. Tsitsonis, ${ }^{47}$ M. Bartók ${ }^{48, t}$ M. Csanad ${ }^{48}$ N. Filipovic, ${ }^{48}$ P. Major ${ }^{48}$ M. I. Nagy, ${ }^{48}$ G. Pasztor ${ }^{48}$ O. Surányi, ${ }^{48}$ G. I. Veres, ${ }^{48}$ G. Bencze,${ }^{49}$ C. Hajdu ${ }^{49}$ D. Horvath,${ }^{49, u}$ Á. Hunyadi ${ }^{49}$ F. Sikler, ${ }^{49}$ T. Á. Vámi, ${ }^{49}$ V. Veszpremi, ${ }^{49}$ G. Vesztergombi ${ }^{49, a, v}$ N. Beni, ${ }^{50}$ S. Czellar, ${ }^{50}$ J. Karancsi, ${ }^{50, t}$ A. Makovec, ${ }^{50}$ J. Molnar, ${ }^{50}$ Z. Szillasi, ${ }^{50}$ P. Raics, ${ }^{51}$ Z. L. Trocsanyi ${ }^{51}$ B. Ujvari, ${ }^{51}$ S. Choudhury, ${ }^{52}$ J. R. Komaragiri, ${ }^{52}$ P. C. Tiwari, ${ }^{52}$ S. Bahinipati,${ }^{53, w}$ C. Kar, ${ }^{53}$ P. Mal,${ }^{53}$ K. Mandal,${ }^{53}$ A. Nayak, ${ }^{53, x}$ D. K. Sahoo, ${ }^{53, w}$ S. K. Swain, ${ }^{53}$ S. Bansal,${ }^{54}$ S. B. Beri, ${ }^{54}$ V. Bhatnagar,${ }^{54}$ S. Chauhan,${ }^{54}$

R. Chawla, ${ }^{54}$ N. Dhingra, ${ }^{54}$ R. Gupta,${ }^{54}$ A. Kaur, ${ }^{54}$ M. Kaur, ${ }^{54}$ S. Kaur, ${ }^{54}$ P. Kumari, ${ }^{54}$ M. Lohan, ${ }^{54}$ A. Mehta,${ }^{54}$ K. Sandeep, ${ }^{54}$ S. Sharma, ${ }^{54}$ J. B. Singh, ${ }^{54}$ A. K. Virdi, ${ }^{54}$ G. Walia, ${ }^{54}$ A. Bhardwaj, ${ }^{55}$ B. C. Choudhary, ${ }^{55}$ R. B. Garg, ${ }^{55}$ M. Gola ${ }^{55}$ S. Keshri, ${ }^{55}$ Ashok Kumar, ${ }^{55}$ S. Malhotra, ${ }^{55}$ M. Naimuddin, ${ }^{55}$ P. Priyanka, ${ }^{55}$ K. Ranjan, ${ }^{55}$ Aashaq Shah, ${ }^{55}$ R. Sharma, ${ }^{55}$ R. Bhardwaj, ${ }^{56, y}$ M. Bharti, ${ }^{56, y}$ R. Bhattacharya,${ }^{56}$ S. Bhattacharya, ${ }^{56}$ U. Bhawandeep, ${ }^{56, y}$ D. Bhowmik, ${ }^{56}$ S. Dey, ${ }^{56}$ S. Dutt, ${ }^{56, y}$ S. Dutta, ${ }^{56}$ S. Ghosh,${ }^{56}$ K. Mondal, ${ }^{56}$ S. Nandan, ${ }^{56}$ A. Purohit, ${ }^{56}$ P. K. Rout, ${ }^{56}$ A. Roy, ${ }^{56}$

S. Roy Chowdhury, ${ }^{56}$ G. Saha, ${ }^{56}$ S. Sarkar, ${ }^{56}$ M. Sharan ${ }^{56}$ B. Singh, ${ }^{56, y}$ S. Thakur, ${ }^{56, y}$ P. K. Behera, ${ }^{57}$ R. Chudasama ${ }^{58}$ D. Dutta, ${ }^{58}$ V. Jha, ${ }^{58}$ V. Kumar, ${ }^{58}$ P. K. Netrakanti, ${ }^{58}$ L. M. Pant, ${ }^{58}$ P. Shukla, ${ }^{58}$ T. Aziz, ${ }^{59}$ M. A. Bhat, ${ }^{59}$ S. Dugad, ${ }^{59}$ G. B. Mohanty ${ }^{59}$ N. Sur, ${ }^{59}$ B. Sutar,${ }^{59}$ Ravindra Kumar Verma, ${ }^{59}$ S. Banerjee, ${ }^{60}$ S. Bhattacharya ${ }^{60}$ S. Chatterjee, ${ }^{60}$ P. Das, ${ }^{60}$ M. Guchait, ${ }^{60}$ Sa. Jain, ${ }^{60}$ S. Karmakar ${ }^{60}$ S. Kumar,${ }^{60}$ M. Maity, ${ }^{60, z}$ G. Majumder ${ }^{60}$ K. Mazumdar, ${ }^{60}$ N. Sahoo, ${ }^{60}$ T. Sarkar, ${ }^{60, z}$ S. Chauhan, ${ }^{61}$ S. Dube,${ }^{61}$ V. Hegde,${ }^{61}$ A. Kapoor ${ }^{61}$ K. Kothekar,${ }^{61}$ S. Pandey, ${ }^{61}$ A. Rane,${ }^{61}$ A. Rastogi,${ }^{61}$ S. Sharma ${ }^{61}$ S. Chenarani, ${ }^{62, a a}$ E. Eskandari Tadavani,${ }^{62}$ S. M. Etesami, ${ }^{62, a a}$ M. Khakzad, ${ }^{62}$ M. Mohammadi Najafabadi, ${ }^{62}$ M. Naseri, ${ }^{62}$ F. Rezaei Hosseinabadi ${ }^{62}$ B. Safarzadeh ${ }^{62, b b}$ M. Zeinali, ${ }^{62}$ M. Felcini, ${ }^{63}$ M. Grunewald, ${ }^{63}$ M. Abbrescia ${ }^{64 a, 64 b}$ C. Calabria, ${ }^{64 a, 64 b}$ A. Colaleo, ${ }^{64 a}$ D. Creanza, ${ }^{64 a, 64 c}$ L. Cristella,,${ }^{64 a, 64 b}$ N. De Filippis, ${ }^{64 a, 64 c}$ M. De Palma, ${ }^{64 a, 64 b}$ A. Di Florio ${ }^{64 a, 64 b}$ F. Errico, ${ }^{64 a, 64 b}$ L. Fiore ${ }^{64 a}$ A. Gelmi, ${ }^{64 a, 64 b}$ G. Iaselli, ${ }^{64 a, 64 c}$ M. Ince, ${ }^{64 a, 64 b}$ S. Lezki, ${ }^{64 a, 64 b}$ G. Maggi ${ }^{64 a, 64 c}$ M. Maggi, ${ }^{64 a}$ G. Miniello, ${ }^{64 a, 64 b}$ S. My, ${ }^{64 a, 64 b}$ S. Nuzzo, ${ }^{64 a, 64 b}$ A. Pompili, ${ }^{64 a, 64 b}$ G. Pugliese, ${ }^{64 a, 64 c}$ R. Radogna, ${ }^{64 a}$ A. Ranieri, ${ }^{64 a}$ G. Selvaggi, ${ }^{64 a, 64 b}$ A. Sharma ${ }^{64 a}$ L. Silvestris, ${ }^{64 a}$ R. Venditti, ${ }^{64 a}$ P. Verwilligen, ${ }^{64 a}$ G. Zito, ${ }^{64 a}$ G. Abbiendi, ${ }^{65 a}$ C. Battilana ${ }^{65 a, 65 b}$ D. Bonacorsi, ${ }^{65 a, 65 b}$ L. Borgonovi ${ }^{65 a, 65 b}$ S. Braibant-Giacomelli, ${ }^{65 a, 65 b}$ R. Campanini, ${ }^{65 a, 65 b}$ P. Capiluppi,${ }^{65 a, 65 b}$ A. Castro, ${ }^{65 a, 65 b}$ F. R. Cavallo, ${ }^{65 a}$ S. S. Chhibra,${ }^{65 a, 65 b}$ C. Ciocca, ${ }^{65 a}$ G. Codispoti ${ }^{65 a, 65 b}$ M. Cuffiani,${ }^{65 a, 65 b}$ G. M. Dallavalle, ${ }^{65 a}$ F. Fabbri, ${ }^{65 a}$ A. Fanfani, ${ }^{65 a, 65 b}$ E. Fontanesi ${ }^{65 a}$ P. Giacomelli, ${ }^{65 a}$ C. Grandi, ${ }^{65 a}$ L. Guiducci, ${ }^{65 a, 65 b}$ F. Iemmi, ${ }^{65 a, 65 b}$ S. Lo Meo, ${ }^{65 a}$ S. Marcellini, ${ }^{65 a}$ G. Masetti, ${ }^{65 a}$ A. Montanari, ${ }^{65 a}$ F. L. Navarria, ${ }^{65 a, 65 b}$ A. Perrotta, ${ }^{65 a}$ F. Primavera, ${ }^{65 a, 65 b, p}$ T. Rovelli, ${ }^{65 a, 65 b}$ G. P. Siroli, ${ }^{65 a, 65 b}$ N. Tosi ${ }^{65 a}$ S. Albergo, ${ }^{66 a, 66 b}$ A. Di Mattia, ${ }^{66 a}$ R. Potenza, ${ }^{66 a, 66 b}$ A. Tricomi ${ }^{66 a, 66 b}$ C. Tuve, ${ }^{66 a, 66 b}$ G. Barbagli ${ }^{67 a}$ K. Chatterjee, ${ }^{67 a, 67 b}$ V. Ciulli, ${ }^{67,67 b}$ C. Civinini, ${ }^{67 a}$ R. D'Alessandro, ${ }^{67 a, 67 b}$ E. Focardi, ${ }^{67,67 b}$ G. Latino, ${ }^{67 a}$ P. Lenzi, ${ }^{67,67 b}$ M. Meschini, ${ }^{67 a}$ S. Paoletti,${ }^{67 a}$ L. Russo, ${ }^{67 a, c c}$ G. Sguazzoni, ${ }^{67 a}$ D. Strom, ${ }^{67 a}$ L. Viliani ${ }^{67 \mathrm{a}}$ L. Benussi, ${ }^{68}$ S. Bianco,${ }^{68}$ F. Fabbri,${ }^{68}$ D. Piccolo ${ }^{68}$ F. Ferro ${ }^{69 a}$ R. Mulargia, ${ }^{69 a, 69 b}$ F. Ravera,${ }^{69 a, 69 b}$

E. Robutti, ${ }^{69 \mathrm{a}}$ S. Tosi ${ }^{69 \mathrm{a}, 69 \mathrm{~b}}$ A. Benaglia, ${ }^{70 \mathrm{a}}$ A. Beschi, ${ }^{70 a, 70 \mathrm{~b}}$ F. Brivio, ${ }^{70 \mathrm{a}, 70 \mathrm{~b}}$ V. Ciriolo, ${ }^{70 \mathrm{a}, 70 \mathrm{~b}, \mathrm{p}} \mathrm{S}$. Di Guida, ${ }^{70 \mathrm{a}, 70 \mathrm{~b}, \mathrm{p}}$ M. E. Dinardo, ${ }^{70 a, 70 b}$ S. Fiorendi, ${ }^{70 a, 70 b}$ S. Gennai, ${ }^{70 a}$ A. Ghezzi, ${ }^{70 a, 70 b}$ P. Govoni, ${ }^{70 a, 70 b}$ M. Malberti, ${ }^{70 a, 70 b}$ S. Malvezzi, ${ }^{70 a}$ A. Massironi, ${ }^{70 a, 70 b}$ D. Menasce, ${ }^{70 a}$ F. Monti, ${ }^{70 a}$ L. Moroni, ${ }^{70 a}$ M. Paganoni, ${ }^{70 a, 70 b}$ D. Pedrini, ${ }^{70 a}$ S. Ragazzi, ${ }^{70 a, 70 b}$ T. Tabarelli de Fatis, ${ }^{70 a, 70 b}$ D. Zuolo, ${ }^{70 a, 70 b}$ S. Buontempo, ${ }^{71 a}$ N. Cavallo, ${ }^{71 a, 71 c}$ A. De Iorio, ${ }^{71 a, 71 b}$ A. Di Crescenzo, ${ }^{71 a, 71 b}$ F. Fabozzi, ${ }^{71 a, 71 \mathrm{c}}$ F. Fienga, ${ }^{71 \mathrm{a}}$ G. Galati, ${ }^{71 \mathrm{a}}$ A. O. M. Iorio, ${ }^{71 \mathrm{a}, 71 \mathrm{~b}}$ W. A. Khan, ${ }^{71 \mathrm{a}}$ L. Lista, ${ }^{71 \mathrm{a}}$ S. Meola, ${ }^{71 a, 71 \mathrm{~d}, \mathrm{p}}$ P. Paolucci, ${ }^{71 a, p}$ C. Sciacca, ${ }^{71 a, 71 b}$ E. Voevodina, ${ }^{71 a, 71 b}$ P. Azzi, ${ }^{72 a}$ N. Bacchetta, ${ }^{72 a}$ A. Boletti, ${ }^{72 a, 72 b}$ A. Bragagnolo, ${ }^{72 a}$ R. Carlin, ${ }^{72 a, 72 b}$ P. Checchia, ${ }^{72 a}$ M. Dall'Osso, ${ }^{72 a, 72 b}$ P. De Castro Manzano, ${ }^{72 a}$ T. Dorigo, ${ }^{72 a}$ U. Dosselli, ${ }^{72 a}$ F. Gasparini, ${ }^{72 a, 72 b}$

U. Gasparini, ${ }^{72 a, 72 b}$ S. Y. Hoh, ${ }^{72 a}$ S. Lacaprara, ${ }^{72 a}$ P. Lujan, ${ }^{72 a}$ M. Margoni, ${ }^{72 a, 72 b}$ A. T. Meneguzzo, ${ }^{72 a, 72 b}$ M. Passaseo, ${ }^{72 a}$ J. Pazzini, ${ }^{72 a, 72 b}$ N. Pozzobon, ${ }^{72 a, 72 b}$ P. Ronchese, ${ }^{72 a, 72 b}$ R. Rossin, ${ }^{72 a, 72 b}$ F. Simonetto, ${ }^{72 a, 72 b}$ A. Tiko, ${ }^{72 a}$ E. Torassa, ${ }^{72 a}$ 
M. Tosi, ${ }^{72 a, 72 b}$ M. Zanetti, ${ }^{72 a, 72 b}$ P. Zotto, ${ }^{72 a, 72 b}$ G. Zumerle, ${ }^{72 a, 72 b}$ A. Braghieri, ${ }^{73 a}$ A. Magnani, ${ }^{73 a}$ P. Montagna, ${ }^{73 a, 73 b}$ S. P. Ratti, ${ }^{73 a, 73 b}$ V. Re, ${ }^{73 a}$ M. Ressegotti, ${ }^{73 a, 73 b}$ C. Riccardi, ${ }^{73 a, 73 b}$ P. Salvini, ${ }^{73 a}$ I. Vai, ${ }^{73 a, 73 b}$ P. Vitulo, ${ }^{73 a, 73 b}$ M. Biasini, ${ }^{74 a, 74 b}$ G. M. Bilei, ${ }^{74 a}$ C. Cecchi, ${ }^{74 a, 74 b}$ D. Ciangottini, ${ }^{74 a, 74 b}$ L. Fanò, ${ }^{74 a, 74 b}$ P. Lariccia, ${ }^{74 a, 74 b}$ R. Leonardi, ${ }^{74 a, 74 b}$ E. Manoni, ${ }^{74 a}$ G. Mantovani, ${ }^{74 a, 74 b}$ V. Mariani, ${ }^{74 a, 74 b}$ M. Menichelli, ${ }^{74 a}$ A. Rossi, ${ }^{74 a, 74 b}$ A. Santocchia, ${ }^{74 a, 74 b}$ D. Spiga,${ }^{74 a}$ K. Androsov, ${ }^{75 a}$ P. Azzurri, ${ }^{75 a}$ G. Bagliesi, ${ }^{75 a}$ L. Bianchini, ${ }^{75 a}$ T. Boccali, ${ }^{75 a}$ L. Borrello, ${ }^{75 a}$ R. Castaldi, ${ }^{75 a}$ M. A. Ciocci $,{ }^{75 a}, 75 b$ R. Dell'Orso, ${ }^{75 a}$ G. Fedi, ${ }^{75 a}$ F. Fiori, ${ }^{75 a, 75 c}$ L. Giannini, ${ }^{75 a, 75 c}$ A. Giassi, ${ }^{75 a}$ M. T. Grippo, ${ }^{75 a}$ F. Ligabue, ${ }^{75 a, 75 c}$ E. Manca, ${ }^{75 a, 75 c}$ G. Mandorli, ${ }^{75 a, 75 c}$ A. Messineo, ${ }^{75 a, 75 b}$ F. Palla, ${ }^{75 a}$ A. Rizzi,${ }^{75 a, 75 b}$ G. Rolandi,${ }^{75 a, d d}$ P. Spagnolo, ${ }^{75 a}$ R. Tenchini,${ }^{75 a}$ G. Tonelli, ${ }^{75 a, 75 b}$ A. Venturi, ${ }^{75 a}$ P. G. Verdini, ${ }^{75 a}$ L. Barone, ${ }^{76 a, 76 b}$ F. Cavallari, ${ }^{76 a}$ M. Cipriani, ${ }^{76 a, 76 b}$ D. Del Re, ${ }^{76 a, 76 b}$ E. Di Marco, ${ }^{76 a, 76 b}$ M. Diemoz, ${ }^{76 a}$ S. Gelli, ${ }^{76 a, 76 b}$ E. Longo, ${ }^{76 a, 76 b}$ B. Marzocchi, ${ }^{76 a, 76 b}$ P. Meridiani, ${ }^{76 a}$ G. Organtini, ${ }^{76 a, 76 b}$ F. Pandolfi, ${ }^{76 a}$ R. Paramatti, ${ }^{76 a, 76 b}$ F. Preiato, ${ }^{76 a, 76 b}$ S. Rahatlou, ${ }^{76 a, 76 b}$ C. Rovelli, ${ }^{76 a}$ F. Santanastasio, ${ }^{76 a, 76 b}$ N. Amapane, ${ }^{77 a, 77 b}$ R. Arcidiacono, ${ }^{77 a, 77 c}$ S. Argiro, ${ }^{77 a, 77 b}$ M. Arneodo, ${ }^{77 a, 77 c}$ N. Bartosik, ${ }^{77 a}$ R. Bellan, ${ }^{77 a, 77 b}$ C. Biino, ${ }^{77 a}$ A. Cappati, ${ }^{77 a, 77 b}$ N. Cartiglia, ${ }^{77 a}$ F. Cenna, ${ }^{77 a, 77 b}$ S. Cometti, ${ }^{77 a}$ M. Costa, ${ }^{77 a, 77 b}$ R. Covarelli, ${ }^{77 a, 77 b}$ N. Demaria, ${ }^{77 a}$ B. Kiani, ${ }^{77 a, 77 b}$ C. Mariotti ${ }^{77 \mathrm{a}}$ S. Maselli, ${ }^{77 \mathrm{a}}$ E. Migliore, ${ }^{77 \mathrm{a}, 77 \mathrm{~b}}$ V. Monaco, ${ }^{77 a, 77 b}$ E. Monteil,${ }^{77 a, 77 b}$ M. Monteno,${ }^{77 a}$ M. M. Obertino, ${ }^{77 a, 77 b}$ L. Pacher, ${ }^{77 a, 77 b}$ N. Pastrone, ${ }^{77 a}$ M. Pelliccioni, ${ }^{77 a}$ G. L. Pinna Angioni, ${ }^{77 a, 77 b}$ A. Romero, ${ }^{77 a, 77 b}$ M. Ruspa,${ }^{77 a, 77 c}$ R. Sacchi, ${ }^{77 a, 77 b}$ R. Salvatico, ${ }^{77 a, 77 b}$ K. Shchelina, ${ }^{77 a, 77 b}$ V. Sola, ${ }^{77 a}$ A. Solano, ${ }^{77 a, 77 b}$ D. Soldi, ${ }^{77 a, 77 b}$ A. Staiano, ${ }^{77 a}$ S. Belforte, ${ }^{78 a}$ V. Candelise, ${ }^{78 a, 78 b}$ M. Casarsa,${ }^{78 a}$ F. Cossutti, ${ }^{78 a}$ A. Da Rold, ${ }^{78 a, 78 b}$ G. Della Ricca, ${ }^{78 a, 78 b}$ F. Vazzoler,${ }^{78 a, 78 b}$ A. Zanetti, ${ }^{78 a}$ D. H. Kim, ${ }^{79}$ G. N. Kim, ${ }^{79}$ M. S. Kim, ${ }^{79}$ J. Lee, ${ }^{79}$ S. Lee, ${ }^{79}$ S. W. Lee, ${ }^{79}$ C. S. Moon, ${ }^{79}$ Y. D. Oh, ${ }^{79}$ S. I. Pak, ${ }^{79}$ S. Sekmen, ${ }^{79}$ D. C. Son, ${ }^{79}$ Y. C. Yang, ${ }^{79}$ H. Kim,${ }^{80}$ D. H. Moon, ${ }^{80}$ G. Oh, ${ }^{80}$ B. Francois, ${ }^{81}$ J. Goh, ${ }^{81, e e ~ T . ~ J . ~ K i m, ~}{ }^{81}$ S. Cho, ${ }^{82}$ S. Choi, ${ }^{82}$ Y. Go, ${ }^{82}$ D. Gyun, ${ }^{82}$ S. Ha, ${ }^{82}$ B. Hong,${ }^{82}$ Y. Jo, ${ }^{82}$ K. Lee, ${ }^{82}$ K. S. Lee, ${ }^{82}$ S. Lee, ${ }^{82}$ J. Lim, ${ }^{82}$ S. K. Park, ${ }^{82}$ Y. Roh,${ }^{82}$ H. S. Kim, ${ }^{83}$ J. Almond, ${ }^{84}$ J. Kim, ${ }^{84}$ J. S. Kim,${ }^{84}$ H. Lee, ${ }^{84}$ K. Lee, ${ }^{84}$ K. Nam, ${ }^{84}$ S. B. Oh, ${ }^{84}$ B. C. Radburn-Smith,${ }^{84}$ S. h. Seo, ${ }^{84}$ U. K. Yang, ${ }^{84}$ H. D. Yoo, ${ }^{84}$ G. B. Yu, ${ }^{84}$ D. Jeon,${ }^{85}$ H. Kim, ${ }^{85}$ J. H. Kim, ${ }^{85}$ J. S. H. Lee, ${ }^{85}$ I. C. Park, ${ }^{85}$ Y. Choi,${ }^{86}$ C. Hwang, ${ }^{86}$ J. Lee, ${ }^{86}$ I. Yu, ${ }^{86}$ V. Dudenas, ${ }^{87}$ A. Juodagalvis, ${ }^{87}$ J. Vaitkus,${ }^{87}$ I. Ahmed, ${ }^{88}$ Z. A. Ibrahim, ${ }^{88}$ M. A. B. Md Ali, ${ }^{88, f f}$ F. Mohamad Idris, ${ }^{88, g g}$ W. A. T. Wan Abdullah, ${ }^{88}$ M. N. Yusli, ${ }^{88}$ Z. Zolkapli, ${ }^{88}$ J. F. Benitez, ${ }^{89}$ A. Castaneda Hernandez ${ }^{89}$ J. A. Murillo Quijada, ${ }^{89}$ H. Castilla-Valdez, ${ }^{90}$ E. De La Cruz-Burelo, ${ }^{90}$ M. C. Duran-Osuna, ${ }^{90}$ I. Heredia-De La Cruz, ${ }^{90, \text { hh }}$ R. Lopez-Fernandez, ${ }^{90}$ J. Mejia Guisao,${ }^{90}$ R. I. Rabadan-Trejo, ${ }^{90}$ M. Ramirez-Garcia, ${ }^{90}$ G. Ramirez-Sanchez, ${ }^{90}$ R. Reyes-Almanza, ${ }^{90}$ A. Sanchez-Hernandez, ${ }^{90}$ S. Carrillo Moreno, ${ }^{91}$ C. Oropeza Barrera, ${ }^{91}$ F. Vazquez Valencia, ${ }^{91}$ J. Eysermans, ${ }^{92}$ I. Pedraza, ${ }^{92}$ H. A. Salazar Ibarguen, ${ }^{92}$ C. Uribe Estrada, ${ }^{92}$ A. Morelos Pineda, ${ }^{93}$ D. Krofcheck, ${ }^{94}$ S. Bheesette, ${ }^{95}$ P. H. Butler, ${ }^{95}$ A. Ahmad, ${ }^{96}$ M. Ahmad, ${ }^{96}$ M. I. Asghar, ${ }^{96}$ Q. Hassan, ${ }^{96}$ H. R. Hoorani, ${ }^{96}$ A. Saddique, ${ }^{96}$ M. A. Shah, ${ }^{96}$ M. Shoaib, ${ }^{96}$ M. Waqas, ${ }^{96}$ H. Bialkowska, ${ }^{97}$ M. Bluj,${ }^{97}$ B. Boimska,${ }^{97}$ T. Frueboes,${ }^{97}$ M. Górski, ${ }^{97}$ M. Kazana, ${ }^{97}$ M. Szleper, ${ }^{97}$ P. Traczyk,${ }^{97}$ P. Zalewski, ${ }^{97}$ K. Bunkowski, ${ }^{98}$ A. Byszuk,${ }^{98, i i}$ K. Doroba ${ }^{98}$ A. Kalinowski,${ }^{98}$ M. Konecki, ${ }^{98}$ J. Krolikowski, ${ }^{98}$ M. Misiura, ${ }^{98}$ M. Olszewski, ${ }^{98}$ A. Pyskir, ${ }^{98}$ M. Walczak,${ }^{98}$ M. Araujo, ${ }^{99}$ P. Bargassa, ${ }^{99}$ C. Beirão Da Cruz E Silva, ${ }^{99}$ A. Di Francesco, ${ }^{99}$ P. Faccioli, ${ }^{99}$ B. Galinhas, ${ }^{99}$ M. Gallinaro, ${ }^{99}$ J. Hollar, ${ }^{99}$ N. Leonardo, ${ }^{99}$ J. Seixas, ${ }^{99}$ G. Strong, ${ }^{99}$ O. Toldaiev, ${ }^{99}$ J. Varela, ${ }^{99}$ S. Afanasiev, ${ }^{100}$ P. Bunin, ${ }^{100}$ M. Gavrilenko, ${ }^{100}$ I. Golutvin, ${ }^{100}$ I. Gorbunov, ${ }^{100}$ A. Kamenev, ${ }^{100}$ V. Karjavine, ${ }^{100}$ A. Lanev, ${ }^{100}$ A. Malakhov, ${ }^{100}$ V. Matveev, ${ }^{100, j j, k k}$ P. Moisenz, ${ }^{100}$ V. Palichik, ${ }^{100}$ V. Perelygin, ${ }^{100}$ S. Shmatov, ${ }^{100}$ S. Shulha, ${ }^{100}$ N. Skatchkov, ${ }^{100}$ V. Smirnov, ${ }^{100}$ N. Voytishin, ${ }^{100}$ A. Zarubin, ${ }^{100}$ V. Golovtsov, ${ }^{101}$ Y. Ivanov, ${ }^{101}$ V. Kim, ${ }^{101,11}$ E. Kuznetsova, ${ }^{101, m m}$ P. Levchenko, ${ }^{101}$ V. Murzin, ${ }^{101}$ V. Oreshkin, ${ }^{101}$ I. Smirnov, ${ }^{101}$ D. Sosnov, ${ }^{101}$ V. Sulimov, ${ }^{101}$ L. Uvarov, ${ }^{101}$ S. Vavilov, ${ }^{101}$ A. Vorobyev, ${ }^{101}$ Yu. Andreev, ${ }^{102}$ A. Dermenev, ${ }^{102}$ S. Gninenko, ${ }^{102}$ N. Golubev, ${ }^{102}$ A. Karneyeu, ${ }^{102}$ M. Kirsanov, ${ }^{102}$ N. Krasnikov, ${ }^{102}$ A. Pashenkov, ${ }^{102}$ D. Tlisov, ${ }^{102}$ A. Toropin, ${ }^{102}$ V. Epshteyn, ${ }^{103}$ V. Gavrilov, ${ }^{103}$ N. Lychkovskaya, ${ }^{103}$ V. Popov, ${ }^{103}$ I. Pozdnyakov, ${ }^{103}$ G. Safronov, ${ }^{103}$ A. Spiridonov, ${ }^{103}$ A. Stepennov, ${ }^{103}$ V. Stolin, ${ }^{103}$ M. Toms, ${ }^{103}$ E. Vlasov, ${ }^{103}$ A. Zhokin, ${ }^{103}$ T. Aushev, ${ }^{104}$ M. Chadeeva, ${ }^{105, n n}$ P. Parygin, ${ }^{105}$ D. Philippov, ${ }^{105}$ S. Polikarpov, ${ }^{105, n n}$ E. Popova, ${ }^{105}$ V. Rusinov, ${ }^{105}$ V. Andreev, ${ }^{106}$ M. Azarkin, ${ }^{106}$ I. Dremin, ${ }^{106, k \mathrm{k}}$ M. Kirakosyan, ${ }^{106}$ A. Terkulov, ${ }^{106}$ A. Baskakov, ${ }^{107}$ A. Belyaev ${ }^{107}$ E. Boos, ${ }^{107}$ V. Bunichev, ${ }^{107}$ M. Dubinin, ${ }^{107,00}$ L. Dudko, ${ }^{107}$ A. Ershov, ${ }^{107}$ A. Gribushin, ${ }^{107}$ V. Klyukhin, ${ }^{107}$ O. Kodolova, ${ }^{107}$ I. Lokhtin, ${ }^{107}$ I. Miagkov, ${ }^{107}$ S. Obraztsov, ${ }^{107}$ S. Petrushanko, ${ }^{107}$ V. Savrin, ${ }^{107}$ A. Barnyakov, ${ }^{108, p p}$ V. Blinov, ${ }^{108, p p}$ T. Dimova, ${ }^{108, p p}$ L. Kardapoltsev, ${ }^{108, p p}$ Y. Skovpen, ${ }^{108, p p}$ I. Azhgirey, ${ }^{109}$ I. Bayshev,${ }^{109}$ S. Bitioukov, ${ }^{109}$ D. Elumakhov, ${ }^{109}$ A. Godizov, ${ }^{109}$ V. Kachanov, ${ }^{109}$ A. Kalinin, ${ }^{109}$ D. Konstantinov, ${ }^{109}$ P. Mandrik, ${ }^{109}$ V. Petrov,${ }^{109}$ R. Ryutin, ${ }^{109}$ S. Slabospitskii, ${ }^{109}$ A. Sobol, ${ }^{109}$ S. Troshin, ${ }^{109}$ N. Tyurin, ${ }^{109}$ A. Uzunian, ${ }^{109}$ A. Volkov, ${ }^{109}$ A. Babaev,${ }^{110}$ S. Baidali, ${ }^{110}$ V. Okhotnikov, ${ }^{110}$ P. Adzic, ${ }^{11, q q}$ P. Cirkovic,${ }^{111}$ D. Devetak, ${ }^{111}$ M. Dordevic, ${ }^{111}$ J. Milosevic, ${ }^{111}$ J. Alcaraz Maestre, ${ }^{112}$ A. Álvarez Fernández, ${ }^{112}$ I. Bachiller, ${ }^{112}$ M. Barrio Luna, ${ }^{112}$ J. A. Brochero Cifuentes, ${ }^{112}$ M. Cerrada, ${ }^{112}$ N. Colino, ${ }^{112}$ B. De La Cruz, ${ }^{112}$ A. Delgado Peris, ${ }^{112}$ 
C. Fernandez Bedoya, ${ }^{112}$ J. P. Fernández Ramos, ${ }^{112}$ J. Flix, ${ }^{112}$ M. C. Fouz, ${ }^{112}$ O. Gonzalez Lopez, ${ }^{112}$ S. Goy Lopez, ${ }^{112}$ J. M. Hernandez, ${ }^{112}$ M. I. Josa, ${ }^{112}$ D. Moran, ${ }^{112}$ A. Pérez-Calero Yzquierdo, ${ }^{112}$ J. Puerta Pelayo, ${ }^{112}$ I. Redondo, ${ }^{112}$

L. Romero, ${ }^{112}$ M. S. Soares, ${ }^{112}$ A. Triossi, ${ }^{112}$ C. Albajar, ${ }^{113}$ J. F. de Trocóniz, ${ }^{113}$ J. Cuevas, ${ }^{114}$ C. Erice,${ }^{114}$

J. Fernandez Menendez, ${ }^{114}$ S. Folgueras, ${ }^{114}$ I. Gonzalez Caballero, ${ }^{114}$ J. R. González Fernández, ${ }^{114}$ E. Palencia Cortezon, ${ }^{114}$ V. Rodríguez Bouza, ${ }^{114}$ S. Sanchez Cruz, ${ }^{114}$ P. Vischia, ${ }^{114}$ J. M. Vizan Garcia, ${ }^{114}$ I. J. Cabrillo, ${ }^{115}$ A. Calderon, ${ }^{115}$

B. Chazin Quero, ${ }^{115}$ J. Duarte Campderros, ${ }^{115}$ M. Fernandez, ${ }^{115}$ P. J. Fernández Manteca, ${ }^{115}$ A. García Alonso, ${ }^{115}$ J. Garcia-Ferrero, ${ }^{115}$ G. Gomez, ${ }^{115}$ A. Lopez Virto, ${ }^{115}$ J. Marco,${ }^{115}$ C. Martinez Rivero, ${ }^{115}$ P. Martinez Ruiz del Arbol, ${ }^{115}$ F. Matorras, ${ }^{115}$ J. Piedra Gomez, ${ }^{115}$ C. Prieels, ${ }^{115}$ T. Rodrigo, ${ }^{115}$ A. Ruiz-Jimeno, ${ }^{115}$ L. Scodellaro, ${ }^{115}$ N. Trevisani, ${ }^{115}$ I. Vila, ${ }^{115}$ R. Vilar Cortabitarte, ${ }^{115}$ N. Wickramage, ${ }^{116}$ D. Abbaneo, ${ }^{117}$ B. Akgun, ${ }^{117}$ E. Auffray, ${ }^{117}$ G. Auzinger, ${ }^{117}$ P. Baillon, ${ }^{117}$ A. H. Ball, ${ }^{117}$ D. Barney, ${ }^{117}$ J. Bendavid, ${ }^{117}$ M. Bianco, ${ }^{117}$ A. Bocci, ${ }^{117}$ C. Botta,${ }^{117}$ E. Brondolin, ${ }^{117}$

T. Camporesi, ${ }^{117}$ M. Cepeda, ${ }^{117}$ G. Cerminara, ${ }^{117}$ E. Chapon, ${ }^{117}$ Y. Chen, ${ }^{117}$ G. Cucciati, ${ }^{117}$ D. d'Enterria, ${ }^{117}$

A. Dabrowski, ${ }^{117}$ N. Daci, ${ }^{117}$ V. Daponte, ${ }^{117}$ A. David,${ }^{117}$ A. De Roeck, ${ }^{117}$ N. Deelen, ${ }^{117}$ M. Dobson, ${ }^{117}$ M. Dünser, ${ }^{117}$ N. Dupont, ${ }^{117}$ A. Elliott-Peisert, ${ }^{117}$ P. Everaerts, ${ }^{117}$ F. Fallavollita, ${ }^{17, \text { rr }}$ D. Fasanella, ${ }^{117}$ G. Franzoni, ${ }^{117}$ J. Fulcher, ${ }^{117}$ W. Funk, ${ }^{117}$ D. Gigi, ${ }^{117}$ A. Gilbert, ${ }^{117}$ K. Gill, ${ }^{117}$ F. Glege, ${ }^{117}$ M. Gruchala, ${ }^{117}$ M. Guilbaud, ${ }^{117}$ D. Gulhan, ${ }^{117}$ J. Hegeman,,${ }^{117}$ C. Heidegger, ${ }^{117}$ V. Innocente, ${ }^{117}$ A. Jafari, ${ }^{117}$ P. Janot,${ }^{117}$ O. Karacheban,,${ }^{17, s}$ J. Kieseler, ${ }^{117}$ A. Kornmayer, ${ }^{117}$

M. Krammer, ${ }^{117, b}$ C. Lange, ${ }^{117}$ P. Lecoq,${ }^{117}$ C. Lourenço, ${ }^{117}$ L. Malgeri, ${ }^{117}$ M. Mannelli, ${ }^{117}$ F. Meijers, ${ }^{117}$ J. A. Merlin, ${ }^{117}$

S. Mersi,${ }^{117}$ E. Meschi,${ }^{117}$ P. Milenovic, ${ }^{117, s s}$ F. Moortgat,${ }^{117}$ M. Mulders, ${ }^{117}$ J. Ngadiuba ${ }^{117}$ S. Nourbakhsh,${ }^{117}$

S. Orfanelli, ${ }^{117}$ L. Orsini, ${ }^{117}$ F. Pantaleo ${ }^{117, p}$ L. Pape ${ }^{117}$ E. Perez, ${ }^{117}$ M. Peruzzi, ${ }^{117}$ A. Petrilli, ${ }^{117}$ G. Petrucciani, ${ }^{117}$ A. Pfeiffer, ${ }^{117}$ M. Pierini, ${ }^{117}$ F. M. Pitters,${ }^{117}$ D. Rabady ${ }^{117}$ A. Racz, ${ }^{117}$ T. Reis, ${ }^{117}$ M. Rovere, ${ }^{117}$ H. Sakulin, ${ }^{117}$ C. Schäfer, ${ }^{117}$ C. Schwick, ${ }^{117}$ M. Seidel, ${ }^{117}$ M. Selvaggi, ${ }^{117}$ A. Sharma, ${ }^{117}$ P. Silva, ${ }^{117}$ P. Sphicas, ${ }^{117, t t}$ A. Stakia, ${ }^{117}$ J. Steggemann, ${ }^{117}$ D. Treille, ${ }^{117}$ A. Tsirou, ${ }^{117}$ V. Veckalns, ${ }^{117, \text { uu }}$ M. Verzetti, ${ }^{117}$ W. D. Zeuner, ${ }^{117}$ L. Caminada, ${ }^{118, v v}$ K. Deiters, ${ }^{118}$ W. Erdmann, ${ }^{118}$ R. Horisberger, ${ }^{118}$ Q. Ingram, ${ }^{118}$ H. C. Kaestli, ${ }^{118}$ D. Kotlinski, ${ }^{118}$ U. Langenegger,${ }^{118}$ T. Rohe, ${ }^{118}$ S. A. Wiederkehr, ${ }^{118}$ M. Backhaus, ${ }^{119}$ L. Bäni, ${ }^{119}$ P. Berger, ${ }^{119}$ N. Chernyavskaya, ${ }^{119}$ G. Dissertori, ${ }^{119}$ M. Dittmar, ${ }^{119}$ M. Donegà ${ }^{119}$ C. Dorfer, ${ }^{119}$ T. A. Gómez Espinosa,${ }^{119}$ C. Grab, ${ }^{119}$ D. Hits, ${ }^{119}$ T. Klijnsma, ${ }^{119}$ W. Lustermann, ${ }^{119}$ R. A. Manzoni, ${ }^{119}$ M. Marionneau, ${ }^{119}$ M. T. Meinhard ${ }^{119}$ F. Micheli, ${ }^{119}$ P. Musella, ${ }^{119}$ F. Nessi-Tedaldi, ${ }^{119}$ J. Pata, ${ }^{119}$ F. Pauss, ${ }^{119}$ G. Perrin, ${ }^{119}$ L. Perrozzi, ${ }^{119}$ S. Pigazzini, ${ }^{119}$ M. Quittnat, ${ }^{119}$ C. Reissel, ${ }^{119}$ D. Ruini, ${ }^{119}$ D. A. Sanz Becerra, ${ }^{119}$ M. Schönenberger ${ }^{119}$ L. Shchutska, ${ }^{119}$ V. R. Tavolaro, ${ }^{119}$ K. Theofilatos, ${ }^{119}$ M. L. Vesterbacka Olsson, ${ }^{119}$ R. Wallny, ${ }^{119}$ D. H. Zhu, ${ }^{119}$ T. K. Aarrestad, ${ }^{120}$ C. Amsler, ${ }^{120, w w}$ D. Brzhechko, ${ }^{120}$ M. F. Canelli, ${ }^{120}$ A. De Cosa, ${ }^{120}$ R. Del Burgo, ${ }^{120}$ S. Donato, ${ }^{120}$ C. Galloni, ${ }^{120}$ T. Hreus, ${ }^{120}$ B. Kilminster, ${ }^{120}$ S. Leontsinis, ${ }^{120}$ I. Neutelings, ${ }^{120}$ G. Rauco, ${ }^{120}$ P. Robmann, ${ }^{120}$ D. Salerno, ${ }^{120}$ K. Schweiger, ${ }^{120}$ C. Seitz, ${ }^{120}$ Y. Takahashi, ${ }^{120}$ A. Zucchetta, ${ }^{120}$ T. H. Doan, ${ }^{121}$ R. Khurana, ${ }^{121}$ C. M. Kuo, ${ }^{121}$ W. Lin, ${ }^{121}$ A. Pozdnyakov, ${ }^{121}$ S. S. Yu, ${ }^{121}$ P. Chang, ${ }^{122}$ Y. Chao, ${ }^{122}$ K. F. Chen, ${ }^{122}$ P. H. Chen, ${ }^{122}$ W.-S. Hou, ${ }^{122}$ Arun Kumar, ${ }^{122}$ Y. F. Liu, ${ }^{122}$ R.-S. Lu, ${ }^{122}$ E. Paganis, ${ }^{122}$ A. Psallidas, ${ }^{122}$ A. Steen, ${ }^{122}$ B. Asavapibhop ${ }^{123}$ N. Srimanobhas, ${ }^{123}$ N. Suwonjandee, ${ }^{123}$ A. Bat, ${ }^{124}$ F. Boran, ${ }^{124}$ S. Cerci, ${ }^{124, x x}$ S. Damarseckin, ${ }^{124}$ Z. S. Demiroglu, ${ }^{124}$ F. Dolek, ${ }^{124}$ C. Dozen, ${ }^{124}$

I. Dumanoglu, ${ }^{124}$ E. Eskut, ${ }^{124}$ S. Girgis, ${ }^{124}$ G. Gokbulut, ${ }^{124}$ Y. Guler, ${ }^{124}$ E. Gurpinar, ${ }^{124}$ I. Hos, ${ }^{124, y y}$ C. Isik, ${ }^{124}$

E. E. Kangal, ${ }^{124, z z}$ O. Kara ${ }^{124}$ A. Kayis Topaksu, ${ }^{124}$ U. Kiminsu, ${ }^{124}$ M. Oglakci, ${ }^{124}$ G. Onengut, ${ }^{124}$ K. Ozdemir, ${ }^{124, \text { aaa }}$ S. Ozturk, ${ }^{124, b b b}$ A. Polatoz, ${ }^{124}$ U. G. Tok, ${ }^{124}$ S. Turkcapar, ${ }^{124}$ I. S. Zorbakir, ${ }^{124}$ C. Zorbilmez, ${ }^{124}$ B. Isildak, ${ }^{125, c c c}$ G. Karapinar, ${ }^{125, \text { ddd }}$ M. Yalvac, ${ }^{125}$ M. Zeyrek, ${ }^{125}$ I. O. Atakisi, ${ }^{126}$ E. Gülmez, ${ }^{126}$ M. Kaya, ${ }^{126, \text { eee }}$ O. Kaya, ${ }^{126, f f f}$

S. Ozkorucuklu, ${ }^{126, g g g}$ S. Tekten, ${ }^{126}$ E. A. Yetkin, ${ }^{126, \text { hhh }}$ M. N. Agaras, ${ }^{127}$ A. Cakir, ${ }^{127}$ K. Cankocak, ${ }^{127}$ Y. Komurcu, ${ }^{127}$ S. Sen, ${ }^{127, \text { iii }}$ B. Grynyov, ${ }^{128}$ L. Levchuk,${ }^{129}$ F. Ball, ${ }^{130}$ J. J. Brooke, ${ }^{130}$ D. Burns, ${ }^{130}$ E. Clement, ${ }^{130}$ D. Cussans, ${ }^{130}$ O. Davignon, ${ }^{130}$ H. Flacher, ${ }^{130}$ J. Goldstein, ${ }^{130}$ G. P. Heath, ${ }^{130}$ H. F. Heath, ${ }^{130}$ L. Kreczko, ${ }^{130}$ D. M. Newbold, ${ }^{130, j j j}$ S. Paramesvaran, ${ }^{130}$ B. Penning, ${ }^{130}$ T. Sakuma, ${ }^{130}$ D. Smith, ${ }^{130}$ V. J. Smith, ${ }^{130}$ J. Taylor, ${ }^{130}$ A. Titterton, ${ }^{130}$ K. W. Bell, ${ }^{131}$ A. Belyaev, ${ }^{131, k \mathrm{kk}}$ C. Brew, ${ }^{131}$ R. M. Brown, ${ }^{131}$ D. Cieri, ${ }^{131}$ D. J. A. Cockerill, ${ }^{131}$ J. A. Coughlan, ${ }^{131}$ K. Harder, ${ }^{131}$ S. Harper ${ }^{131}$ J. Linacre, ${ }^{131}$ E. Olaiya ${ }^{131}$ D. Petyt,${ }^{131}$ C. H. Shepherd-Themistocleous, ${ }^{131}$ A. Thea, ${ }^{131}$ I. R. Tomalin, ${ }^{131}$ T. Williams, ${ }^{131}$ W. J. Womersley, ${ }^{131}$ R. Bainbridge, ${ }^{132}$ P. Bloch, ${ }^{132}$ J. Borg, ${ }^{132}$ S. Breeze, ${ }^{132}$ O. Buchmuller, ${ }^{132}$ A. Bundock, ${ }^{132}$ D. Colling, ${ }^{132}$ P. Dauncey, ${ }^{132}$ G. Davies, ${ }^{132}$ M. Della Negra, ${ }^{132}$ R. Di Maria, ${ }^{132}$ G. Hall, ${ }^{132}$ G. Iles, ${ }^{132}$ T. James, ${ }^{132}$ M. Komm, ${ }^{132}$ C. Laner, ${ }^{132}$ L. Lyons, ${ }^{132}$ A.-M. Magnan, ${ }^{132}$ S. Malik, ${ }^{132}$ A. Martelli, ${ }^{132}$ J. Nash, ${ }^{132,111}$ A. Nikitenko, ${ }^{132, \mathrm{~h}}$ V. Palladino, ${ }^{132}$ M. Pesaresi, ${ }^{132}$ D. M. Raymond, ${ }^{132}$ A. Richards, ${ }^{132}$ A. Rose, ${ }^{132}$ E. Scott, ${ }^{132}$ C. Seez, ${ }^{132}$ A. Shtipliyski, ${ }^{132}$ G. Singh, ${ }^{132}$ M. Stoye, ${ }^{132}$ T. Strebler, ${ }^{132}$ S. Summers, ${ }^{132}$ A. Tapper ${ }^{132}$ K. Uchida ${ }^{132}$ T. Virdee, ${ }^{132, p}$ N. Wardle, ${ }^{132}$ D. Winterbottom, ${ }^{132}$ J. Wright, ${ }^{132}$ S. C. Zenz, ${ }^{132}$ J. E. Cole,${ }^{133}$ P. R. Hobson, ${ }^{133}$ A. Khan, ${ }^{133}$ P. Kyberd, ${ }^{133}$ 
C. K. Mackay, ${ }^{133}$ A. Morton, ${ }^{133}$ I. D. Reid, ${ }^{133}$ L. Teodorescu, ${ }^{133}$ S. Zahid, ${ }^{133}$ K. Call, ${ }^{134}$ J. Dittmann, ${ }^{134}$ K. Hatakeyama, ${ }^{134}$ H. Liu, ${ }^{134}$ C. Madrid, ${ }^{134}$ B. McMaster, ${ }^{134}$ N. Pastika, ${ }^{134}$ C. Smith,,${ }^{134}$ R. Bartek, ${ }^{135}$ A. Dominguez, ${ }^{135}$ A. Buccilli, ${ }^{136}$ S. I. Cooper, ${ }^{136}$ C. Henderson, ${ }^{136}$ P. Rumerio, ${ }^{136}$ C. West, ${ }^{136}$ D. Arcaro, ${ }^{137}$ T. Bose, ${ }^{137}$ D. Gastler, ${ }^{137}$ D. Pinna, ${ }^{137}$ D. Rankin, ${ }^{137}$ C. Richardson, ${ }^{137}$ J. Rohlf, ${ }^{137}$ L. Sulak, ${ }^{137}$ D. Zou,${ }^{137}$ G. Benelli, ${ }^{138}$ X. Coubez, ${ }^{138}$ D. Cutts, ${ }^{138}$ M. Hadley, ${ }^{138}$ J. Hakala, ${ }^{138}$ U. Heintz, ${ }^{138}$ J. M. Hogan, ${ }^{138, \mathrm{mmm}}$ K. H. M. Kwok, ${ }^{138}$ E. Laird, ${ }^{138}$ G. Landsberg, ${ }^{138}$ J. Lee, ${ }^{138}$ Z. Mao, ${ }^{138}$ M. Narain, ${ }^{138}$ S. Sagir, ${ }^{138, n n n}$ R. Syarif, ${ }^{138}$ E. Usai, ${ }^{138}$ D. Yu, ${ }^{138}$ R. Band, ${ }^{139}$ C. Brainerd ${ }^{139}$ R. Breedon, ${ }^{139}$ D. Burns, ${ }^{139}$ M. Calderon De La Barca Sanchez, ${ }^{139}$ M. Chertok, ${ }^{139}$ J. Conway, ${ }^{139}$ R. Conway, ${ }^{139}$ P. T. Cox, ${ }^{139}$ R. Erbacher, ${ }^{139}$ C. Flores, ${ }^{139}$ G. Funk, ${ }^{139}$ W. Ko, ${ }^{139}$ O. Kukral, ${ }^{139}$ R. Lander, ${ }^{139}$ M. Mulhearn, ${ }^{139}$ D. Pellett, ${ }^{139}$ J. Pilot, ${ }^{139}$ S. Shalhout,${ }^{139}$ M. Shi, ${ }^{139}$ D. Stolp, ${ }^{139}$ D. Taylor, ${ }^{139}$ K. Tos, ${ }^{139}$ M. Tripathi, ${ }^{139}$ Z. Wang, ${ }^{139}$ F. Zhang, ${ }^{139}$ M. Bachtis, ${ }^{140}$ C. Bravo, ${ }^{140}$ R. Cousins,${ }^{140}$ A. Dasgupta, ${ }^{140}$ A. Florent, ${ }^{140}$ J. Hauser, ${ }^{140}$ M. Ignatenko, ${ }^{140}$ N. Mccoll,${ }^{140}$ S. Regnard, ${ }^{140}$ D. Saltzberg, ${ }^{140}$ C. Schnaible,${ }^{140}$ V. Valuev, ${ }^{140}$ E. Bouvier, ${ }^{141}$ K. Burt, ${ }^{141}$ R. Clare,${ }^{141}$ J. W. Gary, ${ }^{141}$ S. M. A. Ghiasi Shirazi, ${ }^{141}$ G. Hanson, ${ }^{141}$

G. Karapostoli, ${ }^{141}$ E. Kennedy, ${ }^{141}$ F. Lacroix, ${ }^{141}$ O. R. Long, ${ }^{141}$ M. Olmedo Negrete, ${ }^{141}$ M. I. Paneva, ${ }^{141}$ W. Si, ${ }^{141}$ L. Wang, ${ }^{141}$ H. Wei,${ }^{141}$ S. Wimpenny, ${ }^{141}$ B. R. Yates,${ }^{141}$ J. G. Branson, ${ }^{142}$ P. Chang ${ }^{142}$ S. Cittolin, ${ }^{142}$ M. Derdzinski, ${ }^{142}$ R. Gerosa, ${ }^{142}$ D. Gilbert, ${ }^{142}$ B. Hashemi, ${ }^{142}$ A. Holzner, ${ }^{142}$ D. Klein, ${ }^{142}$ G. Kole, ${ }^{142}$ V. Krutelyov, ${ }^{142}$ J. Letts, ${ }^{142}$ M. Masciovecchio, ${ }^{142}$ D. Olivito, ${ }^{142}$ S. Padhi, ${ }^{142}$ M. Pieri, ${ }^{142}$ M. Sani, ${ }^{142}$ V. Sharma, ${ }^{142}$ S. Simon, ${ }^{142}$ M. Tadel,${ }^{142}$ A. Vartak, ${ }^{142}$ S. Wasserbaech, ${ }^{142, \text { ooo }}$ J. Wood, ${ }^{142}$ F. Würthwein, ${ }^{142}$ A. Yagil, ${ }^{142}$ G. Zevi Della Porta, ${ }^{142}$ N. Amin, ${ }^{143}$ R. Bhandari, ${ }^{143}$ C. Campagnari, ${ }^{143}$ M. Citron, ${ }^{143}$ V. Dutta, ${ }^{143}$ M. Franco Sevilla, ${ }^{143}$ L. Gouskos, ${ }^{143}$ R. Heller, ${ }^{143}$ J. Incandela, ${ }^{143}$ A. Ovcharova, ${ }^{143}$ H. Qu, ${ }^{143}$ J. Richman, ${ }^{143}$ D. Stuart, ${ }^{143}$ I. Suarez, ${ }^{143}$ S. Wang, ${ }^{143}$ J. Yoo, ${ }^{143}$ D. Anderson, ${ }^{144}$ A. Bornheim, ${ }^{144}$ J. M. Lawhorn, ${ }^{144}$ N. Lu, ${ }^{144}$ H. B. Newman, ${ }^{144}$ T. Q. Nguyen, ${ }^{144}$ M. Spiropulu, ${ }^{144}$ J. R. Vlimant, ${ }^{144}$ R. Wilkinson, ${ }^{144}$ S. Xie, ${ }^{144}$ Z. Zhang, ${ }^{144}$ R. Y. Zhu, ${ }^{144}$ M. B. Andrews, ${ }^{145}$ T. Ferguson, ${ }^{145}$ T. Mudholkar, ${ }^{145}$ M. Paulini, ${ }^{145}$ M. Sun, ${ }^{145}$ I. Vorobiev, ${ }^{145}$ M. Weinberg, ${ }^{145}$ J. P. Cumalat, ${ }^{146}$ W. T. Ford, ${ }^{146}$ F. Jensen, ${ }^{146}$ A. Johnson, ${ }^{146}$ E. MacDonald, ${ }^{146}$ T. Mulholland, ${ }^{146}$ R. Patel, ${ }^{146}$ A. Perloff, ${ }^{146}$ K. Stenson, ${ }^{146}$ K. A. Ulmer, ${ }^{146}$ S. R. Wagner, ${ }^{146}$ J. Alexander,${ }^{147}$ J. Chaves, ${ }^{147}$ Y. Cheng, ${ }^{147}$ J. Chu, ${ }^{147}$ A. Datta, ${ }^{147}$ K. Mcdermott, ${ }^{147}$ N. Mirman, ${ }^{147}$ J. R. Patterson, ${ }^{147}$ D. Quach,${ }^{147}$ A. Rinkevicius,${ }^{147}$

A. Ryd,${ }^{147}$ L. Skinnari, ${ }^{147}$ L. Soffi ${ }^{147}$ S. M. Tan, ${ }^{147}$ Z. Tao, ${ }^{147}$ J. Thom, ${ }^{147}$ J. Tucker,${ }^{147}$ P. Wittich, ${ }^{147}$ M. Zientek, ${ }^{147}$

S. Abdullin, ${ }^{148}$ M. Albrow, ${ }^{148}$ M. Alyari, ${ }^{148}$ G. Apollinari, ${ }^{148}$ A. Apresyan, ${ }^{148}$ A. Apyan, ${ }^{148}$ S. Banerjee, ${ }^{148}$

L. A. T. Bauerdick, ${ }^{148}$ A. Beretvas, ${ }^{148}$ J. Berryhill, ${ }^{148}$ P. C. Bhat, ${ }^{148}$ K. Burkett, ${ }^{148}$ J. N. Butler, ${ }^{148}$ A. Canepa, ${ }^{148}$

G. B. Cerati, ${ }^{148}$ H. W. K. Cheung, ${ }^{148}$ F. Chlebana, ${ }^{148}$ M. Cremonesi, ${ }^{148}$ J. Duarte, ${ }^{148}$ V. D. Elvira, ${ }^{148}$ J. Freeman, ${ }^{148}$ Z. Gecse, ${ }^{148}$ E. Gottschalk, ${ }^{148}$ L. Gray, ${ }^{148}$ D. Green, ${ }^{148}$ S. Grünendahl, ${ }^{148}$ O. Gutsche, ${ }^{148}$ J. Hanlon, ${ }^{148}$ R. M. Harris, ${ }^{148}$ S. Hasegawa, ${ }^{148}$ J. Hirschauer, ${ }^{148}$ Z. Hu, ${ }^{148}$ B. Jayatilaka, ${ }^{148}$ S. Jindariani, ${ }^{148}$ M. Johnson, ${ }^{148}$ U. Joshi, ${ }^{148}$ B. Klima, ${ }^{148}$ M. J. Kortelainen, ${ }^{148}$ B. Kreis, ${ }^{148}$ S. Lammel, ${ }^{148}$ D. Lincoln, ${ }^{148}$ R. Lipton, ${ }^{148}$ M. Liu, ${ }^{148}$ T. Liu, ${ }^{148}$ J. Lykken, ${ }^{148}$

K. Maeshima, ${ }^{148}$ J. M. Marraffino, ${ }^{148}$ D. Mason, ${ }^{148}$ P. McBride, ${ }^{148}$ P. Merkel ${ }^{148}$ S. Mrenna, ${ }^{148}$ S. Nahn, ${ }^{148}$ V. O’ Dell, ${ }^{148}$ K. Pedro, ${ }^{148}$ C. Pena, ${ }^{148}$ O. Prokofyev, ${ }^{148}$ G. Rakness, ${ }^{148}$ L. Ristori, ${ }^{148}$ A. Savoy-Navarro, ${ }^{148, p p p}$ B. Schneider, ${ }^{148}$ E. Sexton-Kennedy, ${ }^{148}$ A. Soha, ${ }^{148}$ W. J. Spalding, ${ }^{148}$ L. Spiegel, ${ }^{148}$ S. Stoynev,${ }^{148}$ J. Strait, ${ }^{148}$ N. Strobbe,${ }^{148}$ L. Taylor, ${ }^{148}$ S. Tkaczyk,${ }^{148}$ N. V. Tran, ${ }^{148}$ L. Uplegger,${ }^{148}$ E. W. Vaandering, ${ }^{148}$ C. Vernieri, ${ }^{148}$ M. Verzocchi, ${ }^{148}$ R. Vidal, ${ }^{148}$ M. Wang, ${ }^{148}$ H. A. Weber, ${ }^{148}$ A. Whitbeck, ${ }^{148}$ D. Acosta, ${ }^{149}$ P. Avery, ${ }^{149}$ P. Bortignon, ${ }^{149}$ D. Bourilkov, ${ }^{149}$ A. Brinkerhoff, ${ }^{149}$ L. Cadamuro, ${ }^{149}$ A. Carnes, ${ }^{149}$ D. Curry, ${ }^{149}$ R. D. Field, ${ }^{149}$ S. V. Gleyzer, ${ }^{149}$ B. M. Joshi, ${ }^{149}$ J. Konigsberg, ${ }^{149}$ A. Korytov, ${ }^{149}$ K. H. Lo, ${ }^{149}$ P. Ma, ${ }^{149}$ K. Matchev, ${ }^{149}$ H. Mei,${ }^{149}$ G. Mitselmakher,${ }^{149}$ D. Rosenzweig,${ }^{149}$ K. Shi, ${ }^{149}$ D. Sperka, ${ }^{149}$ J. Wang, ${ }^{149}$ S. Wang, ${ }^{149}$ X. Zuo, ${ }^{149}$ Y. R. Joshi ${ }^{150}$ S. Linn, ${ }^{150}$ A. Ackert, ${ }^{151}$ T. Adams, ${ }^{151}$ A. Askew, ${ }^{151}$ S. Hagopian, ${ }^{151}$ V. Hagopian, ${ }^{151}$ K. F. Johnson, ${ }^{151}$ T. Kolberg, ${ }^{151}$ G. Martinez, ${ }^{151}$ T. Perry, ${ }^{151}$ H. Prosper, ${ }^{151}$ A. Saha, ${ }^{151}$ C. Schiber, ${ }^{151}$ R. Yohay, ${ }^{151}$ M. M. Baarmand, ${ }^{152}$ V. Bhopatkar, ${ }^{152}$ S. Colafranceschi, ${ }^{152}$ M. Hohlmann, ${ }^{152}$ D. Noonan, ${ }^{152}$ M. Rahmani, ${ }^{152}$ T. Roy, ${ }^{152}$ F. Yumiceva, ${ }^{152}$ M. R. Adams, ${ }^{153}$ L. Apanasevich, ${ }^{153}$ D. Berry, ${ }^{153}$ R. R. Betts, ${ }^{153}$ R. Cavanaugh, ${ }^{153}$ X. Chen, ${ }^{153}$ S. Dittmer, ${ }^{153}$ O. Evdokimov, ${ }^{153}$ C. E. Gerber, ${ }^{153}$ D. A. Hangal, ${ }^{153}$ D. J. Hofman, ${ }^{153}$ K. Jung, ${ }^{153}$ J. Kamin, ${ }^{153}$ C. Mills, ${ }^{153}$

I. D. Sandoval Gonzalez, ${ }^{153}$ M. B. Tonjes, ${ }^{153}$ H. Trauger, ${ }^{153}$ N. Varelas, ${ }^{153}$ H. Wang, ${ }^{153}$ X. Wang, ${ }^{153}$ Z. Wu, ${ }^{153}$ J. Zhang, ${ }^{153}$ M. Alhusseini, ${ }^{154}$ B. Bilki, ${ }^{154, q q q}$ W. Clarida, ${ }^{154}$ K. Dilsiz, ${ }^{154, \text { rrr }}$ S. Durgut, ${ }^{154}$ R. P. Gandrajula, ${ }^{154}$ M. Haytmyradov, ${ }^{154}$ V. Khristenko, ${ }^{154}$ J.-P. Merlo, ${ }^{154}$ A. Mestvirishvili, ${ }^{154}$ A. Moeller, ${ }^{154}$ J. Nachtman, ${ }^{154}$ H. Ogul, ${ }^{154, s s s}$ Y. Onel,${ }^{154}$ F. Ozok, ${ }^{154, \text { ttt }}$ A. Penzo, ${ }^{154}$ C. Snyder, ${ }^{154}$ E. Tiras, ${ }^{154}$ J. Wetzel, ${ }^{154}$ B. Blumenfeld, ${ }^{155}$ A. Cocoros,${ }^{155}$ N. Eminizer, ${ }^{155}$ D. Fehling, ${ }^{155}$ L. Feng, ${ }^{155}$ A. V. Gritsan, ${ }^{155}$ W. T. Hung, ${ }^{155}$ P. Maksimovic,,${ }^{155}$ J. Roskes, ${ }^{155}$ U. Sarica, ${ }^{155}$ M. Swartz, ${ }^{155}$ M. Xiao, ${ }^{155}$ C. You, ${ }^{155}$ A. Al-bataineh, ${ }^{156}$ P. Baringer ${ }^{156}$ A. Bean, ${ }^{156}$ S. Boren, ${ }^{156}$ J. Bowen, ${ }^{156}$ A. Bylinkin, ${ }^{156}$ J. Castle, ${ }^{156}$ S. Khalil, ${ }^{156}$ A. Kropivnitskaya, ${ }^{156}$ D. Majumder, ${ }^{156}$ W. Mcbrayer, ${ }^{156}$ M. Murray, ${ }^{156}$ C. Rogan, ${ }^{156}$ S. Sanders, ${ }^{156}$ E. Schmitz, ${ }^{156}$ 
J. D. Tapia Takaki, ${ }^{156}$ Q. Wang, ${ }^{156}$ S. Duric,${ }^{157}$ A. Ivanov ${ }^{157}$ K. Kaadze,${ }^{157}$ D. Kim, ${ }^{157}$ Y. Maravin, ${ }^{157}$ D. R. Mendis, ${ }^{157}$ T. Mitchell, ${ }^{157}$ A. Modak, ${ }^{157}$ A. Mohammadi, ${ }^{157}$ L. K. Saini, ${ }^{157}$ F. Rebassoo, ${ }^{158}$ D. Wright, ${ }^{158}$ A. Baden, ${ }^{159}$ O. Baron, ${ }^{159}$ A. Belloni, ${ }^{159}$ S. C. Eno, ${ }^{159}$ Y. Feng, ${ }^{159}$ C. Ferraioli, ${ }^{159}$ N. J. Hadley, ${ }^{159}$ S. Jabeen, ${ }^{159}$ G. Y. Jeng, ${ }^{159}$ R. G. Kellogg, ${ }^{159}$ J. Kunkle, ${ }^{159}$ A. C. Mignerey, ${ }^{159}$ S. Nabili, ${ }^{159}$ F. Ricci-Tam, ${ }^{159}$ Y. H. Shin, ${ }^{159}$ A. Skuja, ${ }^{159}$ S. C. Tonwar, ${ }^{159}$ K. Wong,${ }^{159}$ D. Abercrombie, ${ }^{160}$ B. Allen, ${ }^{160}$ V. Azzolini, ${ }^{160}$ A. Baty, ${ }^{160}$ G. Bauer, ${ }^{160}$ R. Bi, ${ }^{160}$ S. Brandt, ${ }^{160}$ W. Busza, ${ }^{160}$ I. A. Cali, ${ }^{160}$ M. D'Alfonso, ${ }^{160}$ Z. Demiragli, ${ }^{160}$ G. Gomez Ceballos, ${ }^{160}$ M. Goncharov,${ }^{160}$ P. Harris, ${ }^{160}$ D. Hsu, ${ }^{160}$ M. Hu, ${ }^{160}$ Y. Iiyama, ${ }^{160}$ G. M. Innocenti ${ }^{160}$ M. Klute, ${ }^{160}$ D. Kovalskyi ${ }^{160}$ Y.-J. Lee, ${ }^{160}$ P. D. Luckey, ${ }^{160}$ B. Maier, ${ }^{160}$ A. C. Marini, ${ }^{160}$ C. Mcginn, ${ }^{160}$ C. Mironov, ${ }^{160}$ S. Narayanan, ${ }^{160}$ X. Niu, ${ }^{160}$ C. Paus, ${ }^{160}$ C. Roland, ${ }^{160}$ G. Roland, ${ }^{160}$ Z. Shi, ${ }^{160}$ G. S. F. Stephans, ${ }^{160}$ K. Sumorok, ${ }^{160}$ K. Tatar, ${ }^{160}$ D. Velicanu, ${ }^{160}$ J. Wang, ${ }^{160}$ T. W. Wang, ${ }^{160}$ B. Wyslouch, ${ }^{160}$ A. C. Benvenuti, ${ }^{161, a}$ R. M. Chatterjee, ${ }^{161}$ A. Evans, ${ }^{161}$ P. Hansen, ${ }^{161}$ J. Hiltbrand, ${ }^{161}$ Sh. Jain, ${ }^{161}$ S. Kalafut, ${ }^{161}$ M. Krohn, ${ }^{161}$ Y. Kubota, ${ }^{161}$ Z. Lesko, ${ }^{161}$ J. Mans, ${ }^{161}$ N. Ruckstuhl, ${ }^{161}$ R. Rusack, ${ }^{161}$ M. A. Wadud, ${ }^{161}$ J. G. Acosta, ${ }^{162}$ S. Oliveros, ${ }^{162}$ E. Avdeeva, ${ }^{163}$ K. Bloom, ${ }^{163}$ D. R. Claes, ${ }^{163}$ C. Fangmeier, ${ }^{163}$ F. Golf, ${ }^{163}$ R. Gonzalez Suarez, ${ }^{163}$ R. Kamalieddin, ${ }^{163}$ I. Kravchenko, ${ }^{163}$ J. Monroy, ${ }^{163}$ J. E. Siado, ${ }^{163}$ G. R. Snow, ${ }^{163}$ B. Stieger,${ }^{163}$ A. Godshalk, ${ }^{164}$ C. Harrington, ${ }^{164}$ I. Iashvili, ${ }^{164}$ A. Kharchilava, ${ }^{164}$ C. Mclean, ${ }^{164}$ D. Nguyen, ${ }^{164}$ A. Parker, ${ }^{164}$ S. Rappoccio, ${ }^{164}$ B. Roozbahani, ${ }^{164}$ G. Alverson, ${ }^{165}$ E. Barberis, ${ }^{165}$ C. Freer, ${ }^{165}$ Y. Haddad, ${ }^{165}$ A. Hortiangtham, ${ }^{165}$ D. M. Morse ${ }^{165}$ T. Orimoto, ${ }^{165}$ R. Teixeira De Lima, ${ }^{165}$ T. Wamorkar, ${ }^{165}$ B. Wang, ${ }^{165}$ A. Wisecarver, ${ }^{165}$ D. Wood, ${ }^{165}$ S. Bhattacharya, ${ }^{166}$ J. Bueghly, ${ }^{166}$ O. Charaf, ${ }^{166}$ K. A. Hahn, ${ }^{166}$ N. Mucia, ${ }^{166}$ N. Odell, ${ }^{166}$ M. H. Schmitt, ${ }^{166}$ K. Sung, ${ }^{166}$ M. Trovato, ${ }^{166}$ M. Velasco, ${ }^{166}$ R. Bucci, ${ }^{167}$ N. Dev, ${ }^{167}$ M. Hildreth, ${ }^{167}$ K. Hurtado Anampa, ${ }^{167}$ C. Jessop, ${ }^{167}$ D. J. Karmgard, ${ }^{167}$ N. Kellams, ${ }^{167}$ K. Lannon, ${ }^{167}$ W. Li, ${ }^{167}$ N. Loukas, ${ }^{167}$ N. Marinelli, ${ }^{167}$ F. Meng, ${ }^{167}$ C. Mueller, ${ }^{167}$ Y. Musienko, ${ }^{167, j j}$ M. Planer,${ }^{167}$ A. Reinsvold, ${ }^{167}$ R. Ruchti, ${ }^{167}$ P. Siddireddy, ${ }^{167}$ G. Smith,${ }^{167}$ S. Taroni, ${ }^{167}$ M. Wayne, ${ }^{167}$ A. Wightman, ${ }^{167}$ M. Wolf, ${ }^{167}$ A. Woodard, ${ }^{167}$ J. Alimena, ${ }^{168}$ L. Antonelli, ${ }^{168}$ B. Bylsma, ${ }^{168}$ L. S. Durkin, ${ }^{168}$ S. Flowers, ${ }^{168}$ B. Francis, ${ }^{168}$ C. Hill, ${ }^{168}$ W. Ji ${ }^{168}$ T. Y. Ling, ${ }^{168}$ W. Luo, ${ }^{168}$ B. L. Winer,${ }^{168}$ S. Cooperstein,${ }^{169}$ P. Elmer, ${ }^{169}$ J. Hardenbrook, ${ }^{169}$ S. Higginbotham, ${ }^{169}$ A. Kalogeropoulos, ${ }^{169}$ D. Lange, ${ }^{169}$ M. T. Lucchini, ${ }^{169}$ J. Luo, ${ }^{169}$ D. Marlow, ${ }^{169}$ K. Mei, ${ }^{169}$ I. Ojalvo, ${ }^{169}$ J. Olsen, ${ }^{169}$ C. Palmer, ${ }^{169}$ P. Piroué, ${ }^{169}$ J. Salfeld-Nebgen, ${ }^{169}$ D. Stickland, ${ }^{169}$ C. Tully, ${ }^{169}$ Z. Wang, ${ }^{169}$ S. Malik, ${ }^{170}$ S. Norberg, ${ }^{170}$ A. Barker, ${ }^{171}$ V. E. Barnes, ${ }^{171}$ S. Das, ${ }^{171}$ L. Gutay,${ }^{171}$ M. Jones, ${ }^{171}$ A. W. Jung, ${ }^{171}$ A. Khatiwada, ${ }^{171}$ B. Mahakud, ${ }^{171}$ D. H. Miller,${ }^{171}$ N. Neumeister, ${ }^{171}$ C. C. Peng, ${ }^{171}$ S. Piperov,${ }^{171}$ H. Qiu, ${ }^{171}$ J. F. Schulte, ${ }^{171}$ J. Sun, ${ }^{171}$ F. Wang, ${ }^{171}$ R. Xiao, ${ }^{171}$ W. Xie, ${ }^{171}$ T. Cheng, ${ }^{172}$ J. Dolen, ${ }^{172}$ N. Parashar, ${ }^{172}$ Z. Chen, ${ }^{173}$ K. M. Ecklund, ${ }^{173}$ S. Freed ${ }^{173}$ F. J. M. Geurts, ${ }^{173}$ M. Kilpatrick, ${ }^{173}$ W. Li, ${ }^{173}$ B. P. Padley, ${ }^{173}$ R. Redjimi, ${ }^{173}$ J. Roberts, ${ }^{173}$ J. Rorie, ${ }^{173}$ W. Shi, ${ }^{173}$ Z. Tu, ${ }^{173}$ A. Zhang, ${ }^{173}$ A. Bodek, ${ }^{174}$ P. de Barbaro, ${ }^{174}$ R. Demina, ${ }^{174}$ Y. t. Duh, ${ }^{174}$ J. L. Dulemba, ${ }^{174}$ C. Fallon, ${ }^{174}$ T. Ferbel, ${ }^{174}$ M. Galanti, ${ }^{174}$ A. Garcia-Bellido, ${ }^{174}$ J. Han, ${ }^{174}$ O. Hindrichs, ${ }^{174}$ A. Khukhunaishvili, ${ }^{174}$ E. Ranken, ${ }^{174}$ P. Tan, ${ }^{174}$ R. Taus, ${ }^{174}$ A. Agapitos, ${ }^{175}$ J. P. Chou, ${ }^{175}$ Y. Gershtein, ${ }^{175}$ E. Halkiadakis, ${ }^{175}$ A. Hart, ${ }^{175}$ M. Heindl, ${ }^{175}$ E. Hughes, ${ }^{175}$ S. Kaplan, ${ }^{175}$ R. Kunnawalkam Elayavalli, ${ }^{175}$ S. Kyriacou, ${ }^{175}$ A. Lath, ${ }^{175}$ R. Montalvo, ${ }^{175}$ K. Nash,${ }^{175}$ M. Osherson, ${ }^{175}$ H. Saka, ${ }^{175}$ S. Salur, ${ }^{175}$ S. Schnetzer, ${ }^{175}$ D. Sheffield, ${ }^{175}$ S. Somalwar, ${ }^{175}$ R. Stone, ${ }^{175}$ S. Thomas, ${ }^{175}$ P. Thomassen, ${ }^{175}$ M. Walker, ${ }^{175}$ A. G. Delannoy, ${ }^{176}$ J. Heideman, ${ }^{176}$ G. Riley, ${ }^{176}$ S. Spanier, ${ }^{176}$ O. Bouhali, ${ }^{177, \text { uuu }}$ A. Celik, ${ }^{177}$ M. Dalchenko, ${ }^{177}$ M. De Mattia, ${ }^{177}$ A. Delgado, ${ }^{177}$ S. Dildick, ${ }^{177}$ R. Eusebi, ${ }^{177}$ J. Gilmore, ${ }^{177}$ T. Huang, ${ }^{177}$ T. Kamon, ${ }^{177, v v v}$ S. Luo, ${ }^{177}$ R. Mueller ${ }^{177}$ D. Overton, ${ }^{177}$ L. Pernie,${ }^{177}$ D. Rathjens, ${ }^{177}$ A. Safonov, ${ }^{177}$ N. Akchurin, ${ }^{178}$ J. Damgov, ${ }^{178}$ F. De Guio, ${ }^{178}$ P. R. Dudero, ${ }^{178}$ S. Kunori, ${ }^{178}$ K. Lamichhane, ${ }^{178}$ S. W. Lee, ${ }^{178}$ T. Mengke, ${ }^{178}$ S. Muthumuni, ${ }^{178}$ T. Peltola, ${ }^{178}$ S. Undleeb, ${ }^{178}$ I. Volobouev, ${ }^{178}$ Z. Wang, ${ }^{178}$ S. Greene, ${ }^{179}$ A. Gurrola,${ }^{179}$ R. Janjam, ${ }^{179}$ W. Johns, ${ }^{179}$ C. Maguire, ${ }^{179}$ A. Melo, ${ }^{179}$ H. Ni, ${ }^{179}$ K. Padeken, ${ }^{179}$ J. D. Ruiz Alvarez, ${ }^{179}$ P. Sheldon, ${ }^{179}$ S. Tuo, ${ }^{179}$ J. Velkovska, ${ }^{179}$ M. Verweij,,${ }^{179}$ Q. Xu, ${ }^{179}$ M. W. Arenton, ${ }^{180}$ P. Barria, ${ }^{180}$ B. Cox,${ }^{180}$ R. Hirosky, ${ }^{180}$ M. Joyce, ${ }^{180}$ A. Ledovskoy,${ }^{180}$ H. Li ${ }^{180}$ C. Neu, ${ }^{180}$ T. Sinthuprasith ${ }^{180}$ Y. Wang, ${ }^{180}$ E. Wolfe, ${ }^{180}$ F. Xia, ${ }^{180}$ R. Harr, ${ }^{181}$ P. E. Karchin,${ }^{181}$ N. Poudyal, ${ }^{181}$ J. Sturdy, ${ }^{181}$ P. Thapa, ${ }^{181}$ S. Zaleski, ${ }^{181}$ M. Brodski, ${ }^{182}$ J. Buchanan, ${ }^{182}$ C. Caillol, ${ }^{182}$ D. Carlsmith, ${ }^{182}$ S. Dasu, ${ }^{182}$ I. De Bruyn, ${ }^{182}$ L. Dodd ${ }^{182}$ B. Gomber, ${ }^{182}$ M. Grothe, ${ }^{182}$ M. Herndon, ${ }^{182}$ A. Hervé, ${ }^{182}$ U. Hussain, ${ }^{182}$ P. Klabbers, ${ }^{182}$ A. Lanaro, ${ }^{182}$ K. Long, ${ }^{182}$ R. Loveless, ${ }^{182}$ T. Ruggles, ${ }^{182}$ A. Savin, ${ }^{182}$ V. Sharma, ${ }^{182}$ N. Smith, ${ }^{182}$ W. H. Smith, ${ }^{182}$ and N. Woods ${ }^{182}$

(CMS Collaboration)

${ }^{1}$ Yerevan Physics Institute, Yerevan, Armenia

${ }^{2}$ Institut für Hochenergiephysik, Wien, Austria

${ }^{3}$ Institute for Nuclear Problems, Minsk, Belarus 


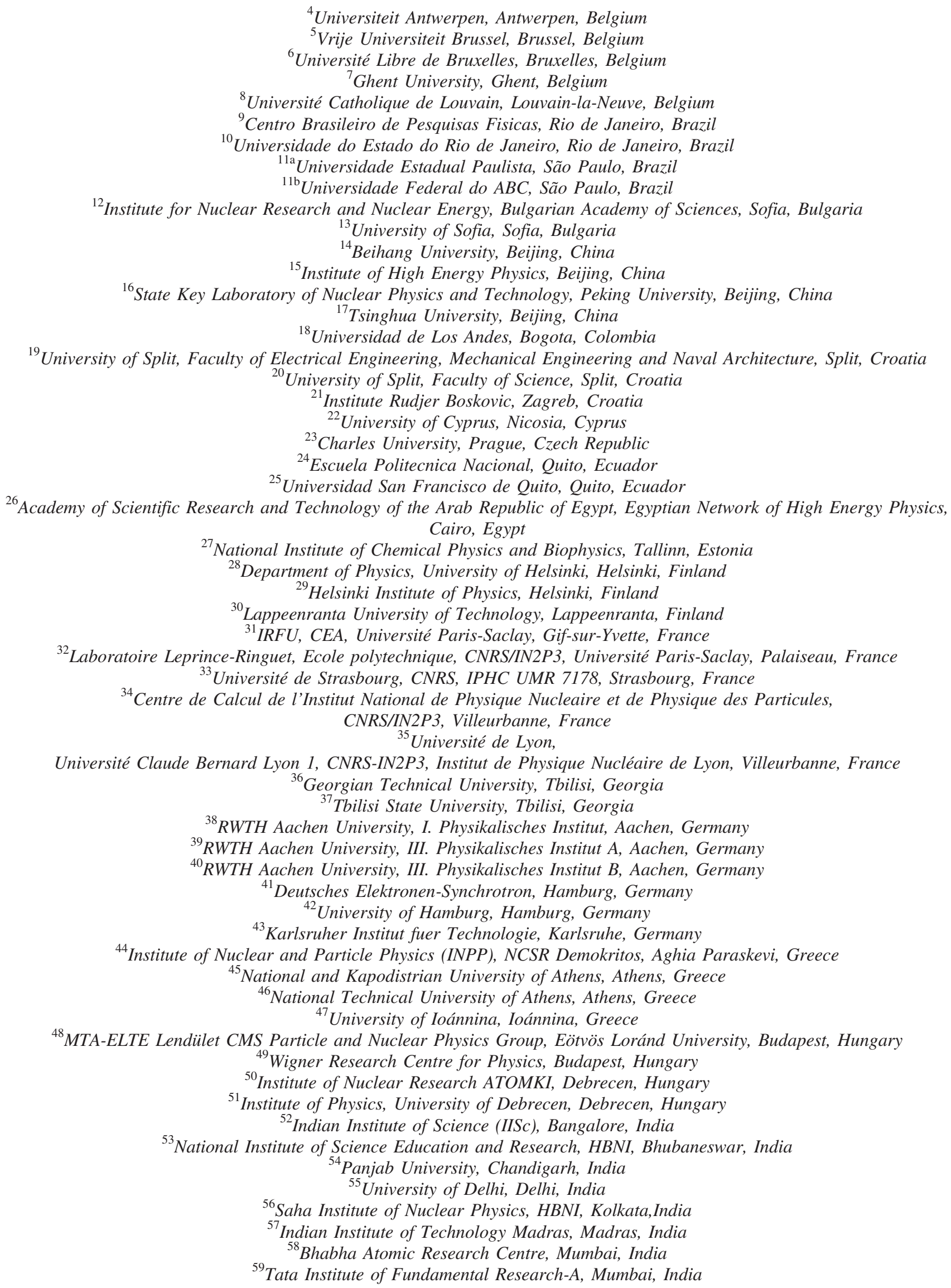




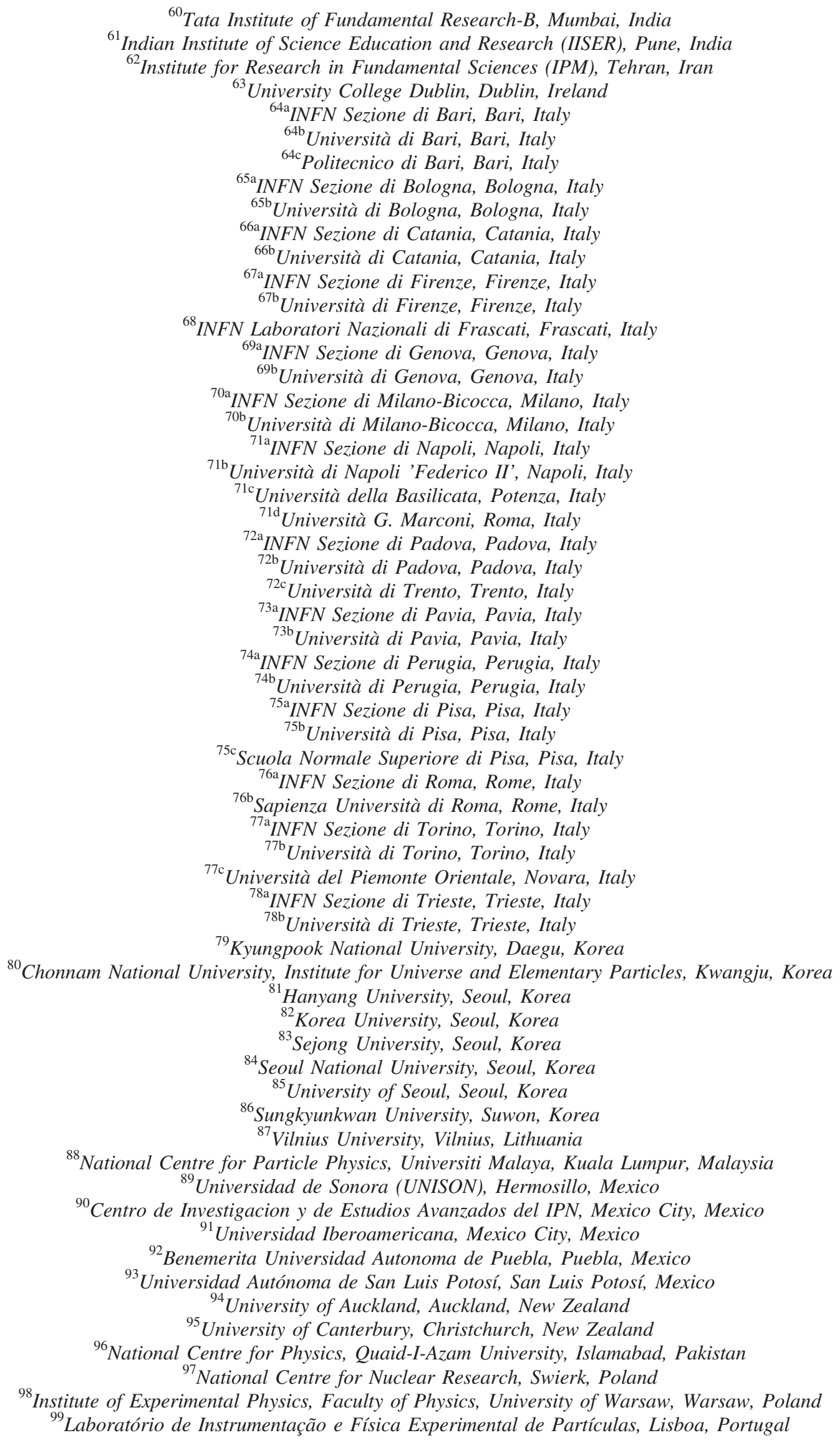




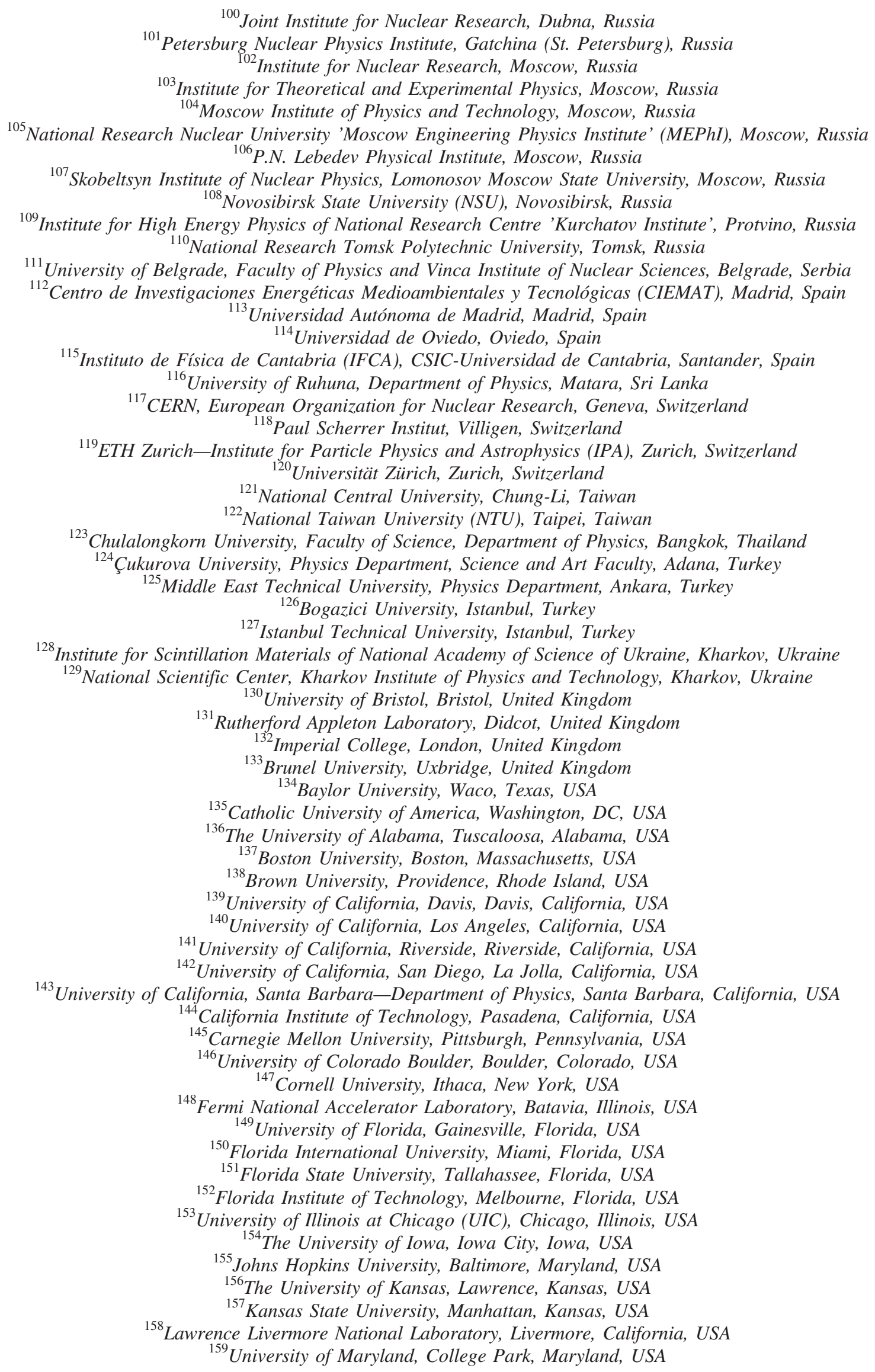




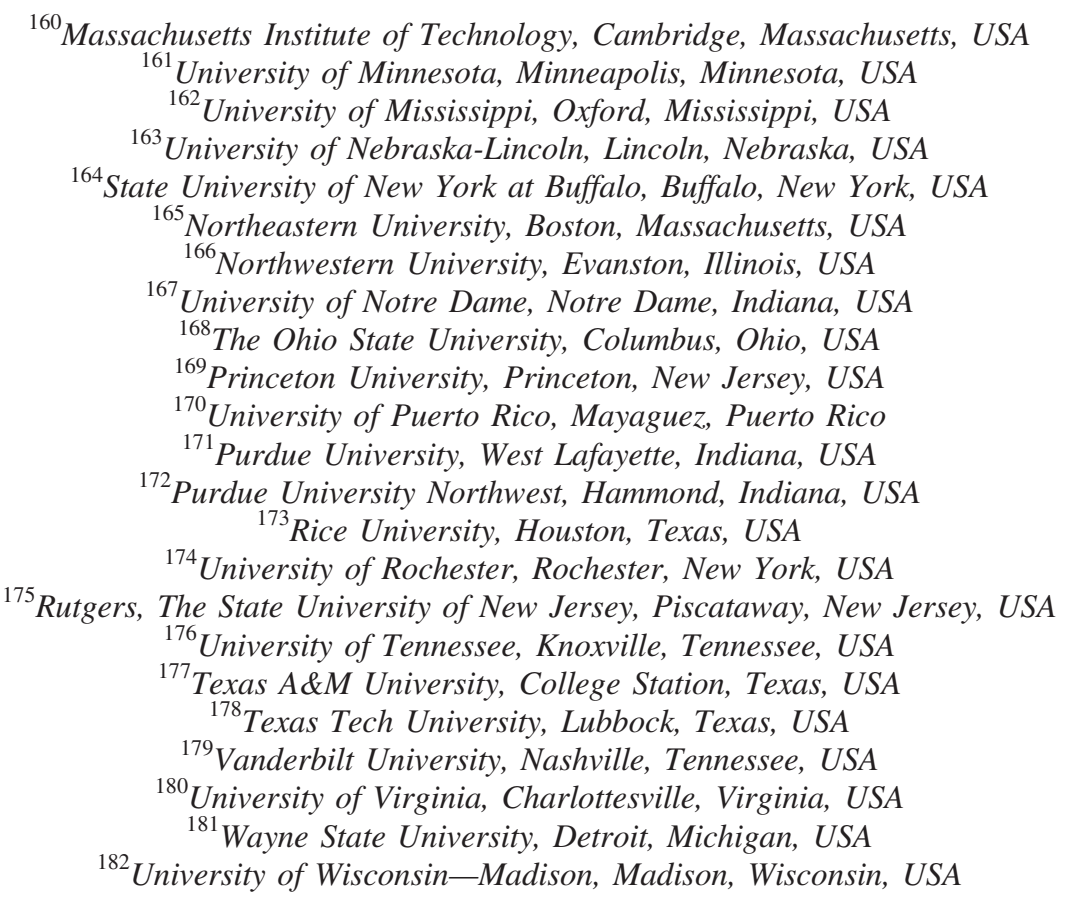

${ }^{\mathrm{a}}$ Deceased.

${ }^{\mathrm{b}}$ Also at Vienna University of Technology, Vienna, Austria.

${ }^{c}$ Also at IRFU, CEA, Université Paris-Saclay, Gif-sur-Yvette, France.

${ }^{\mathrm{d}}$ Also at Universidade Estadual de Campinas, Campinas, Brazil.

${ }^{\mathrm{e}}$ Also at Federal University of Rio Grande do Sul, Porto Alegre, Brazil.

${ }^{\mathrm{f}}$ Also at Université Libre de Bruxelles, Bruxelles, Belgium.

${ }^{g}$ Also at University of Chinese Academy of Sciences, Beijing, China.

${ }^{\mathrm{h}}$ Also at Institute for Theoretical and Experimental Physics, Moscow, Russia.

${ }^{\mathrm{i}}$ Also at Joint Institute for Nuclear Research, Dubna, Russia.

${ }^{\mathrm{j}}$ Also at Cairo University, Cairo, Egypt.

${ }^{\mathrm{k}}$ Also at Helwan University, Cairo, Egypt.

${ }^{1}$ Also at Zewail City of Science and Technology, Zewail, Egypt.

${ }^{\mathrm{m}}$ Also at Department of Physics, King Abdulaziz University, Jeddah, Saudi Arabia.

${ }^{\mathrm{n}}$ Also at Université de Haute Alsace, Mulhouse, France.

${ }^{\circ}$ Also at Skobeltsyn Institute of Nuclear Physics, Lomonosov Moscow State University, Moscow, Russia.

${ }^{\mathrm{p}}$ Also at CERN, European Organization for Nuclear Research, Geneva, Switzerland.

${ }^{\mathrm{q}}$ Also at RWTH Aachen University, III. Physikalisches Institut A, Aachen, Germany.

${ }^{\mathrm{r}}$ Also at University of Hamburg, Hamburg, Germany.

${ }^{\mathrm{s}}$ Also at Brandenburg University of Technology, Cottbus, Germany.

${ }^{t}$ Also at Institute of Physics, University of Debrecen, Debrecen, Hungary.

${ }^{u}$ Also at Institute of Nuclear Research ATOMKI, Debrecen, Hungary.

${ }^{v}$ Also at MTA-ELTE Lendület CMS Particle and Nuclear Physics Group, Eötvös Loránd University, Budapest, Hungary.

${ }^{\mathrm{w}}$ Also at IIT Bhubaneswar, Bhubaneswar, India.

${ }^{\mathrm{x}}$ Also at Institute of Physics, Bhubaneswar, India.

${ }^{\mathrm{y}}$ Also at Shoolini University, Solan, India.

${ }^{\mathrm{z}}$ Also at University of Visva-Bharati, Santiniketan, India.

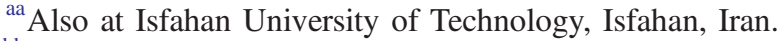

${ }^{\mathrm{bb}}$ Also at Plasma Physics Research Center, Science and Research Branch, Islamic Azad University, Tehran, Iran.

${ }^{c c}$ Also at Università degli Studi di Siena, Siena, Italy.

${ }^{\mathrm{dd}}$ Also at Scuola Normale e Sezione dell'INFN, Pisa, Italy.

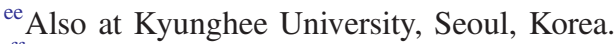

${ }^{\mathrm{ff}}$ Also at International Islamic University of Malaysia, Kuala Lumpur, Malaysia.

${ }^{\mathrm{gg}}$ Also at Malaysian Nuclear Agency, MOSTI, Kajang, Malaysia.

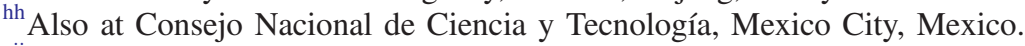

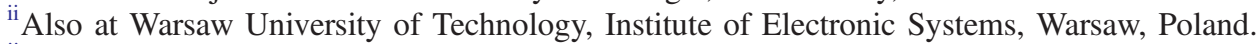

${ }^{\mathrm{jj}}$ Also at Institute for Nuclear Research, Moscow, Russia. 


\footnotetext{
${ }^{\mathrm{kk}}$ Also at National Research Nuclear University 'Moscow Engineering Physics Institute' (MEPhI), Moscow, Russia.

${ }^{11}$ Also at St. Petersburg State Polytechnical University, St. Petersburg, Russia.

${ }^{\mathrm{mm}}$ Also at University of Florida, Gainesville, Florida, USA.

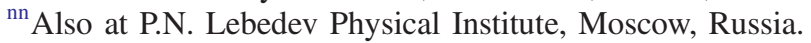

${ }^{\circ o}$ Also at California Institute of Technology, Pasadena, California, USA.

${ }^{\mathrm{pp}}$ Also at Budker Institute of Nuclear Physics, Novosibirsk, Russia.

${ }^{\mathrm{qq}}$ Also at Faculty of Physics, University of Belgrade, Belgrade, Serbia.

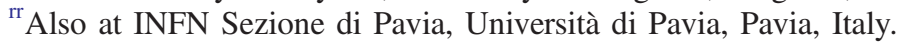

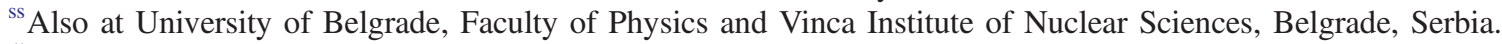

${ }^{t t}$ Also at National and Kapodistrian University of Athens, Athens, Greece.

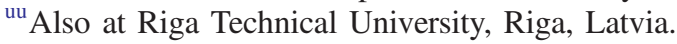

${ }^{\mathrm{vv}}$ Also at Universität Zürich, Zurich, Switzerland.

${ }^{w w}$ Also at Stefan Meyer Institute for Subatomic Physics, Vienna, Austria.

${ }^{\mathrm{xx}}$ Also at Adiyaman University, Adiyaman, Turkey.

${ }^{\text {yy }}$ Also at Istanbul Aydin University, Istanbul, Turkey.

${ }^{\mathrm{zz}}$ Also at Mersin University, Mersin, Turkey.

${ }^{\text {aaa }}$ Also at Piri Reis University, Istanbul, Turkey.

${ }^{b b b}$ Also at Gaziosmanpasa University, Tokat, Turkey.

${ }^{c c c}$ Also at Ozyegin University, Istanbul, Turkey.

${ }^{\text {ddd }}$ Also at Izmir Institute of Technology, Izmir, Turkey.

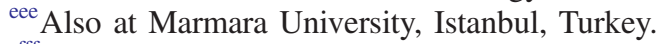

${ }^{\mathrm{fff}}$ Also at Kafkas University, Kars, Turkey.

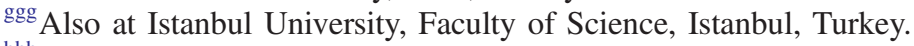

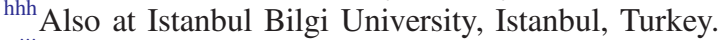

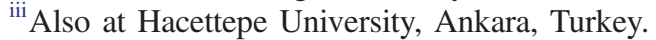

${ }_{\mathrm{jij}}$ Also at Rutherford Appleton Laboratory, Didcot, United Kingdom.

${ }^{k k k}$ Also at School of Physics and Astronomy, University of Southampton, Southampton, United Kingdom.

${ }^{111}$ Also at Monash University, Faculty of Science, Clayton, Australia.

${ }^{\mathrm{mmm}}$ Also at Bethel University, St. Paul, Minneapolis, Minnesota, USA.

${ }^{n n n}$ Also at Karamanoğlu Mehmetbey University, Karaman, Turkey.

${ }^{000}$ Also at Utah Valley University, Orem, Utah, USA.

${ }^{\mathrm{ppp}}$ Also at Purdue University, West Lafayette, Indiana, USA.

${ }^{\mathrm{qqq}}$ Also at Beykent University, Istanbul, Turkey.

${ }^{\mathrm{rrr}}$ Also at Bingol University, Bingol, Turkey.

${ }^{\text {sss }}$ Also at Sinop University, Sinop, Turkey.

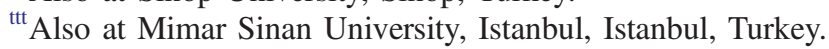

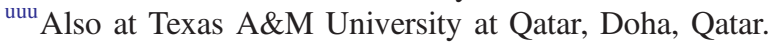

${ }^{\mathrm{vvv}}$ Also at Kyungpook National University, Daegu, Korea.
} 